\title{
SAPT: LIGAÇÃO DE HIDROGÊNIO OU INTERAÇÃO DE van der WAALS?
}

\author{
Boaz G. Oliveira* \\ Instituto de Ciências Ambientais e Desenvolvimento Sustentável, Universidade Federal da Bahia, 47801-100 Barreiras - BA, Brasil \\ Regiane C. M. U. Araújo \\ Departamento de Química, Universidade Federal da Paraíba, 58059-900 João Pessoa - PB, Brasil
}

Recebido em 24/11/11; aceito em 27/6/12; publicado na web em 17/9/12

\begin{abstract}
SAPT: HYDROGEN BOND OR van der WAALS INTERACTION? It is through the application of an electronic partition approach called Symmetry-Adapted Perturbation Theory (SAPT) that the nature of hydrogen bonds and van der Waals interactions can be unveiled according to the contribution of electrostatic, charge transfer, exchange repulsion, polarization, and dispersion terms. Among these, electrostatic partition governs the formation of the hydrogen bonds, whose energies are arguably high. However, the weakness of the interaction strength is caused by dispersion forces, whose contribution decisively lead to the stabilization of complexes formed via van der Waals interactions.
\end{abstract}

Keywords: hydrogen bonds; van der Waals interactions; SAPT.

\section{INTRODUÇÃO}

Fisicamente, as interações fortes, fracas, eletromagnéticas e gravitacionais governam o contato entre as partículas. ${ }^{1}$ Destas, é bem estabelecido pela cromodinâmica quântica que as interações fortes ocorrem entre prótons e nêutrons através da ação dos quarks e glúons, ${ }^{2}$ cujo resultado é a formação do núcleo atômico denso e compacto. Presente em nível nuclear, as interações fracas são $10^{13}$ menos intensas e são caracterizadas pelas manifestações dos bósons W (Weak nuclear force) e Z (Zero). ${ }^{3}$ Interações eletromagnéticas são típicas de partículas elementares carregadas ${ }^{4}$ e são $10^{2}$ mais fracas em relação às interações fortes. Por fim, a geometrodinâmica estabelece que as interações gravitacionais são $10^{42}$ mais amenas e estão presentes em sistemas com massas macroscopicamente mensuráveis, ${ }^{5}$ como estrelas, galáxias e todo o universo. No cerne da química atômico-molecular, as interações consideradas fracas com características puramente eletrostáticas são as mais importantes, pois os sistemas são interpretados como sendo ligados ${ }^{6,7}$ e não covalentes, ${ }^{8,9}$ e nestas circunstâncias átomos formam as ligações químicas ${ }^{10}$ e moléculas atingem condições eletrônicas propícias para formarem estruturas intermoleculares estáveis. ${ }^{11}$

$\mathrm{Na}$ natureza há uma imensidade de sistemas estabilizados intermolecularmente, destes aqueles contendo regiões com altas densidades eletrônicas (pares de elétrons não compartilhados, ${ }^{12-14}$ elétrons $\pi,{ }^{15-17}$ ou hidretos ${ }^{18-21}$ representados por $\mathrm{Y}^{-\delta}$ na ligação $\mathrm{X}-\mathrm{Y}^{-\delta}$ ) são atraídos por espécies doadoras de prótons (ácidos de Lewis ${ }^{22,23}$ simbolizados por $\mathrm{H}^{+\delta}-\mathrm{Z}$ ). O resultado desta atração é uma interação vastamente conhecida como ligação de hidrogênio $\left(\mathrm{X}-\mathrm{Y}^{-\delta} \ldots \mathrm{H}^{+\delta}-\mathrm{Z}\right) \cdot{ }^{24}$

$$
\mathrm{X}-\mathrm{Y}+\mathrm{H}-\mathrm{Z} \rightarrow \mathrm{X}-\mathrm{Y}^{-\delta} \cdot \cdots \mathrm{H}^{+\delta}-\mathrm{Z}
$$

Embora a ligação de hidrogênio já tenha sido reconhecida pela IUPAC, ${ }^{25}$ o conhecimento teórico relacionado à essência fenomenológica desta interação já data de muitos anos. Podem-se citar os estudos empíricos de Latimer e Rodebush, ${ }^{26}$ Kasper, ${ }^{27}$ Coulson e Danielson, ${ }^{28}$ Tsubomura, ${ }^{29}$ Nukasawa et al.,${ }^{30}$ Koski, ${ }^{31}$ Kollman, ${ }^{32}$ até as abordagens mais modernas de Koch e Popelier, ${ }^{33}$ Hobza, ${ }^{34}$ Del Bene $^{35}$ e Grabowski, ${ }^{36}$ os quais através da utilização de campos de

\footnotetext{
*e-mail: boazgaldino@gmail.com
}

força específicos ${ }^{37}$ e/ou métodos de química quântica ${ }^{38-40}$ conduzem a inebriante busca pelo estado da arte intermolecular, decorrente do contato entre um hidrogênio e um sítio molecular com concentração de carga relativamente elevada.

Apesar do conhecimento das propriedades teóricas das ligações de hidrogênio, ${ }^{41}$ outras importantes interações como as ligações de di-hidrogênio ${ }^{42}$ ligações de hidreto ${ }^{43}$ ligações agósticas, ${ }^{44}$ ligações de halogênio, ${ }^{45}$ ligações halogênio-hidreto ${ }^{46}$ e stacking ${ }^{47,48}$ foram consideradas por Lipkowski et al. ${ }^{49}$ como parte integrante do conjunto de ligações intermoleculares mais moderno até então registrado na literatura. Sabe-se que estas interações atingem um grau de abrangência bastante inusitado. Por exemplo, Sándorfy e outros ${ }^{50}$ alertam que o desenvolvimento da medicina perioperatória, relacionado à elucidação do mecanismo de anestesia no sistema nervoso central, está estritamente relacionado ao conhecimento das propriedades das ligações de hidrogênio. Braun e Smirnov ${ }^{51}$ mostraram que os parâmetros espectroscópicos das ligações de hidrogênio justificam o fenômeno da coloração azulada predominante nas águas do Caribe e Mar Mediterrâneo. Por outro lado, a articulação e locomoção de répteis arboríferos, como também a constituição de corpos celestes, podem ser explicadas através de forças típicas das interações de van der Waals, conforme destacado por Autumn et al, ${ }^{52}$ Fernandez e Jockers ${ }^{53}$ e Whipple e Huebner. ${ }^{53}$ As interações intituladas de van der Waals foram assim concebidas em reconhecimento ao físico neerlândes Johannes Diederik van der Waals pelos seus estudos com substâncias gasosas e líquidas, em uma proposta que objetivava a otimização dos modelos de Boyle e Charles ${ }^{54}$ através da descrição do tamanho molecular e a força intermolecular atuante, a qual é composta pelas contribuições de Keesom (interação entre dipolo permanente-dipolo permanente), ${ }^{55}$ Debye (interação entre dipolo permanente-dipolo induzido) ${ }^{56}$ e London (interação entre dipolo induzido-dipolo induzido). ${ }^{57}$ As interações de van der Waals têm sido foco de muitos pesquisas, principalmente no aprimoramento de alguns métodos que falham na captura dos efeitos provenientes das forças de dispersão. ${ }^{58}$

As ligações de hidrogênio ou interações de van der Waals são, de fato, alicerces de suma importância para a Biologia, Medicina, Química, Engenharia, Física ${ }^{59}$ e áreas correlatas. Entretanto, dentro da comunidade científica ainda persiste um intenso debate sobre a seguinte questão: "O que diferencia uma ligação de hidrogênio de uma interação do tipo van der Waals?" ${ }^{60-63}$ Em outras palavras: "Poderia 
o modelo clássico da ligação de hidrogênio $\left(\mathrm{X}-\mathrm{Y}^{-\delta} \ldots \mathrm{H}^{+\delta}-\mathrm{Z}\right)$ também coexistir em uma interação do tipo van der Waals?" De acordo com a proposta de Bian $^{64}$ utilizada para medir a força da ligação de hidrogênio, e nos argumentos de Hammerum ${ }^{65}$ sobre a utilização de radicais alquila como receptores de prótons, é incontestável que a natureza das espécies X-Y e H-Z promova não somente a formação da supermolécula, mas que também seja um guia para investigar a intensidade de interação. No que diz respeito à estabilidade intermolecular, a interpretação das energias potenciais de atração e repulsão existentes entre $\mathrm{X}-\mathrm{Y}$ e $\mathrm{H}-\mathrm{Z}$ inegavelmente conduz a um discernimento mais coerente sobre a interação reinante, conforme ilustra a Figura 1. ${ }^{66}$

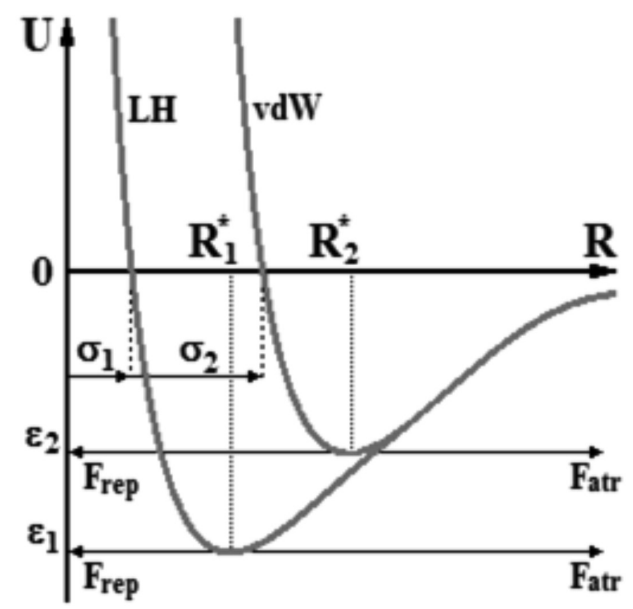

Figura 1. Curvas de energia potencial para ligação de hidrogênio $(\boldsymbol{L H}) e$ interação de van der Waals (vdW $)^{66,68}$

Pode ser constatado que a atração $\left(\mathrm{F}_{\text {atr }}\right)$ intermolecular é única e condicionada a uma maior estabilidade. Por outro lado, a repulsão $\left(\mathrm{F}_{\text {rep }}\right)$ distintamente perfila a ligação de hidrogênio (LH) da interação de van der Waals (vdW), tanto em função da distância interatômica $\left(\sigma_{1}<\sigma_{2}\right)$, como em relação ao mínimo de energia $\left(\varepsilon_{1}<\varepsilon_{2}\right) \cdot{ }^{67}$ Pela descrição de Lennard-Jones para a curva de energia potencial, na Equação $2^{68}$ tem-se os valores para a profundidade do potencial $(\varepsilon)$, para a distância $(\sigma)$ onde o potencial interpartícula é nulo, e que o termo repulsivo apresenta um valor do expoente $n$ igual a 12 para as interações de van der Waals, enquanto nas ligações de hidrogênio o valor de $n$ varia entre 8 e $10 .{ }^{69}$ Devido a isso, as energias das ligações de hidrogênio são mais elevadas em relação às interações de van der Waals. ${ }^{70}$

$$
U=\varepsilon\left[\left(\frac{R^{*}}{R}\right)^{n}-2\left(\frac{R^{*}}{R}\right)^{6}\right]=4 \varepsilon\left[\left(\frac{\sigma}{R}\right)^{n}-\left(\frac{\sigma}{R}\right)^{6}\right]
$$

Gleiter e c-autores ${ }^{71}$ discutiram este raciocínio em um estudo sobre a capacidade dos calcogênios formarem interações intermoleculares atípicas. Algumas destes podem ser observadas nos complexos de selênio $\mathrm{H}_{2} \mathrm{Se}$ - $\mathrm{SeH}_{2}$ (a), $\mathrm{H}_{2} \mathrm{Se} \cdots \mathrm{SeHC}_{2} \mathrm{H}$ (b) e $\mathrm{H}_{2} \mathrm{Se} \cdots \mathrm{SeHCN}$ (c). As estruturas destes sistemas foram caracterizadas em um mínimo da superfície de energia potencial e apresentam a seguinte ordem de estabilidade (a) $<$ (b) $<$ (c) (Figura 2), cujos respectivos valores das energias de interação $\Delta \mathrm{E}$ e distâncias intermoleculares $\mathrm{R}_{(\mathrm{Se} \cdots \mathrm{Se})}$ são $-2,82 \mathrm{kcal} / \mathrm{mol}$ e $3,91 \AA,-3,66 \mathrm{kcal} / \mathrm{mol}$ e $3,63 \AA$, e $-4,62 \mathrm{kcal} / \mathrm{mol}$ e 2,50 ̊. Pelo fato destas interações serem consideradas bastante fracas, Gleiter e seus colaboradores ${ }^{71}$ demonstraram que a estabilização dos sistemas (a), (b) e (c) ocorre com prevalência das forças de dispersão e, sendo assim, a interação $\mathrm{H}-\mathrm{Se} \cdots \mathrm{Se}-\mathrm{R}\left(\mathrm{R}=\mathrm{H}_{2}, \mathrm{H}_{2} \mathrm{C}_{2} \mathrm{e}\right.$ HCN) seguiria o modelo de van der Waals ao invés de uma "ligação de calcogênio". Baseado neste relato, tornou-se conclusivo que o conhecimento da natureza física dos sistemas químicos é a maneira mais adequada para caracterizar o tipo de interação intermolecular existente, mas independentemente do exemplo $\mathrm{H}-\mathrm{Se} \cdots \mathrm{Se}-\mathrm{R}$, a maioria dos estudos envolvendo sistemas intermoleculares é baseada na elucidação dos fenômencos provenientes da formação de ligações de hidrogênio ou interações de van der Waals. Por esta visão, a clássica teoria da eletronegatividade de Pauling ${ }^{72}$ e as ideias de Pimentel e McClellan $^{72}$ já não sustentam a credibilidade da análise não covalente para o modelo $\mathrm{X}-\mathrm{Y}^{-\delta} \ldots \mathrm{H}^{+\delta}-\mathrm{Z} .{ }^{66,73-74}$

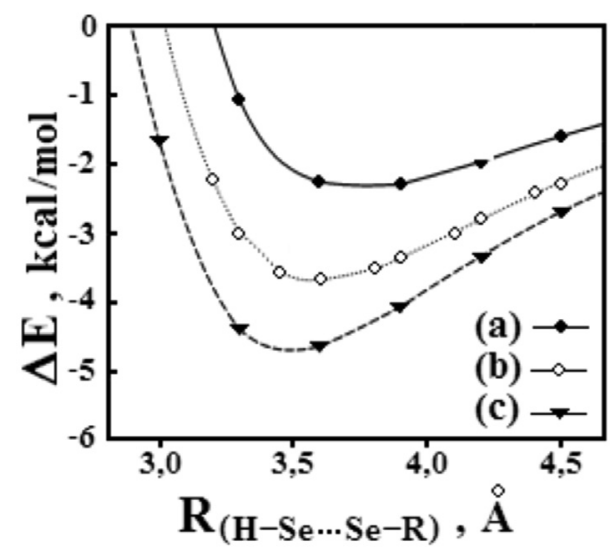

Figura 2. Energias de interação dos complexos $\mathrm{H}_{2} \mathrm{Se} \cdots \mathrm{SeH}_{2}($ a $), \mathrm{H}_{2} \mathrm{Se} \cdots \mathrm{SeHC} \mathrm{S}_{2} \mathrm{H}$ (b) e $\mathrm{H}_{2} \mathrm{Se} \cdots \mathrm{SeHCN}$ (c) obtidas pelo nível de cálculo MP2/cc-pVTZ-ECP. Reproduzida da ref. 71, com permissão da American Chemical Society

Do ponto de vista estrutural, as interações de van de Waals são caracterizadas mediante a análise de raios atômicos tabulados, pelos quais são fornecidos valores de distâncias intermoleculares muito maiores do que as ligações de hidrogênio. Por exemplo, considerando os respectivos valores de 1,20 e 1,52 Å para hidrogênio e oxigênio, ${ }^{75} \mathrm{a}$ distância de van der Waals para a interação $\mathrm{H}$... O apresenta um valor

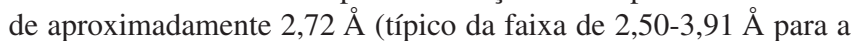
ligação $\mathrm{H}-\mathrm{Se} \cdots \mathrm{Se}-\mathrm{R}) .{ }^{71}$ Entretanto, valores menores compreendidos entre 1,6 e $2,2 \AA$ são obtidos se H...O for interpretada como uma ligação de hidrogênio. Como não há critério físico para este tipo de abordagem estrutural, a diferença entre ligação de hidrogênio e interações de van der Waals requer uma abordagem mais intimista dos termos existenciais que refletem a estabilização do sistema químico. ${ }^{76,77}$

Em 1967, Duijneveldt e Murrell ${ }^{78}$ apresentaram um estudo detalhado sobre os parâmetros que devem ser considerados na formação de um sistema intermolecular. De forma conclusiva, mostraram que a estabilização da ligação de hidrogênio $\mathrm{X}-\mathrm{Y}^{-\delta} \cdots \mathrm{H}^{+\delta}-\mathrm{Z}$ está diretamente vinculada a um potencial intermolecular $U$ descrito pela Equação $3:^{79}$

$$
\mathrm{U}=\mathrm{E}_{\mathrm{C}}+\mathrm{E}_{\mathrm{Ex}}+\mathrm{E}_{\mathrm{Pol}}+\mathrm{E}_{\mathrm{TC}}+\mathrm{E}_{\mathrm{D}}
$$

onde: ${ }^{80} \mathrm{E}_{\mathrm{C}}$ - energia eletrostática de natureza atrativa ou repulsiva; $\mathrm{E}_{\mathrm{Ex}}$ - energia da repulsão entre os spins orbitais na região overlap; $\mathrm{E}_{\mathrm{Pol}}$ - energia de distorção dipolo-dipolo induzido da distribuição eletrônica; $\mathrm{E}_{\mathrm{TC}}$ - energia derivada da transferência de carga entre os orbitais de fronteira e, $E_{D}$ - energia proveniente das forças de dispersão de London.

Como vastamente conhecido, sistemas apolares como benzeno sofrem distorção eletrônica em sua estrutura ocasionada pelo contato com espécies iônicas, conforme ilustrado nas Figuras 3 e 4, pelas quais Sinnokrot, Hohenstein e Sherrill ${ }^{81}$ mostraram que a indução e o efeito eletrostático são os responsáveis pela estabilização entre o benzeno e o cátion sódio, quando a interação é lateral $\mathrm{C}_{6} \mathrm{H}_{6} \cdots \mathrm{Na}^{+}$ou 


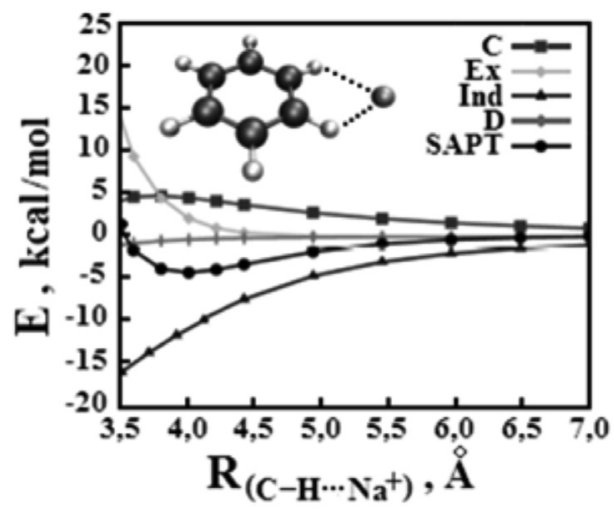

Figura 3. Decomposição SAPTO para o complexo lateral $\mathrm{C}_{6} \mathrm{H}_{6} \cdots \mathrm{Na}^{+}$. Reproduzida da ref. 81, com permissão de John Wiley and Sons

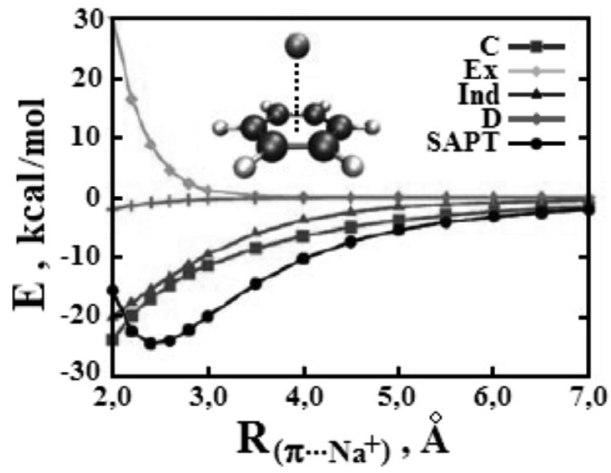

Figura 4. Decomposição SAPTO para o complexo T-shaped $\mathrm{C}_{6} \mathrm{H}_{6} \cdots \mathrm{Na}^{+}$. Reproduzida da ref. 81, com permissão de John Wiley and Sons

na região dos orbitais $\pi\left(\mathrm{C}_{6} \mathrm{H}_{6}\right) \pi \cdots \mathrm{Na}^{+}$, respectivamente.

Em uma comparação com complexos de hidrogênio similares, ${ }_{16,17,82-84}$ é indiscutível que a contribuição eletrostática é o termo dominante na formação das interações intermoleculares ${ }^{85,86}$ Contudo, deve ser sempre mencionado que, como as interações de van der Waals apresentam energias mais baixas em comparação com as ligações de hidrogênio, nestas circunstâncias as forças de dispersão se apresentam como sendo dominantes ${ }^{87}$ conforme já demonstrado para o complexos $\mathrm{H}_{2} \mathrm{Se} \cdots \mathrm{SeH}_{2}$ (a), $\mathrm{H}_{2} \mathrm{Se} \cdots \mathrm{SeHC}_{2} \mathrm{H}$ (b) e $\mathrm{H}_{2} \mathrm{Se} \cdots \mathrm{SeHCN}$ (c) (Figura 2), ${ }^{71}$ mas também observado nos dímeros $\mathrm{CH}_{4} \cdots \mathrm{CH}_{4},{ }^{81} \mathrm{CH}_{4} \cdots \mathrm{HX}(\mathrm{X}$

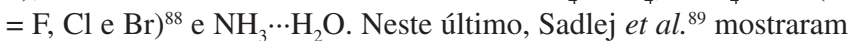
que, embora as forças de dispersão não sejam majoritárias, estas correspondem a aproximadamente $30 \%$ da energia de indução que juntamente com a eletrostática são as responsáveis pela estabilidade intermolecular. Outros trabalhos ressaltam a significativa contribuição da transferência de carga entre os orbitais de fronteira das moléculas $\mathrm{X}-\mathrm{Y}$ (fonte de elétrons) e H-Z (vacância de elétrons) ${ }^{90-92}$ mas o caráter resultante da interação formada ainda é considerado eletrostático com algumas evidências de que a covalência também esteja presente. ${ }^{93}$ De forma generalizada, pela contribuição dos termos $\mathrm{E}_{\mathrm{C}}, \mathrm{E}_{\mathrm{Ex}}, \mathrm{E}_{\mathrm{Pol}}$, $\mathrm{E}_{\mathrm{D}}$ e $\mathrm{E}_{\mathrm{TC}}$ para a força de interação de um determinado sistema intermolecular pode-se afirmar se este é estabilizado através de ligações de hidrogênio (termo coulômbico dominante) ${ }^{94}$ ou interações de van der Waals (termo dispersivo dominante) ${ }^{95}$

\section{MÉTODOS TEÓRICOS DE DECOMPOSIÇÃO DE ENERGIA}

Com o objetivo de elucidar a origem eletrônica das interações intermoleculares através da quantificação dos termos $\mathrm{E}_{\mathrm{C}}, \mathrm{E}_{\mathrm{Ex}}, \mathrm{E}_{\mathrm{Pol}}$, $\mathrm{E}_{\mathrm{D}}$ e $_{\mathrm{TC}}{ }^{78}$ na década de 70 , Morokuma e Umeyama ${ }^{85}$ apresentaram um método denominado de Decomposição de Carga e Densidade Eletrônica (ECDD, Electronic Charge and Density Decomposition) ${ }^{85}$ Extremamente útil no estudo dos dímeros $\mathrm{HF} \cdots \mathrm{HF}, \mathrm{H}_{2} \mathrm{O} \cdots \mathrm{H}_{2} \mathrm{O}$, $\mathrm{NH}_{3} \cdots \mathrm{HF}, \mathrm{LiF} \cdots \mathrm{LiF}$ e $\mathrm{LiH} \cdots \mathrm{LiH}$, os resultados obtidos pela ECDD serviram para entender a "origem da ligação de hidrogênio" e mostraram que a densidade eletrônica na região vicinal do doador de próton diminui $(+\delta=$ vacância de elétrons) devido a uma repulsão originária do potencial de troca de spin (Ex) e da indução (Ind). Conforme claramente argumentado por Hobza et al. ${ }^{96}$ a transferência de carga (TC) entre os orbitais de fronteira HOMO e LUMO das respectivas espécies doadora e receptora de prótons envolve uma repulsão entre seus elétrons na região de overlap intermolecular e a polarização é mais branda por se tratar de uma contribuição entre multipolos induzidos.$^{95}$ De fato, ocorre um aumento $(-\delta=$ fonte de elétrons) da densidade eletrônica na ligação H-Z ocasionado pela indução (Ind) e transferência de carga (TC), a qual se mostra como o segundo termo de atração mais importante, visto que a contribuição coulômbica torna a ligação de hidrogênio um fenômeno essencialmente eletrostático. ${ }^{85}$ Todavia, mesmo a polarização não sendo um componente atrativo tenaz, sua contribuição para a redistribuição de carga é latente.

Entrementes, a descrição analítica dos termos $\mathrm{E}_{\mathrm{C}}, \mathrm{E}_{\mathrm{Ex}}, \mathrm{E}_{\mathrm{Pol}}, \mathrm{E}_{\mathrm{D}} \mathrm{e}$ $\mathrm{E}_{\mathrm{TC}}$ requer cuidado especial com relação à correlação eletrônica, ${ }^{97}$ pois nesta situação as forças de dispersão exercem influência vital na energia total de sistemas fracamente ligados como, por exemplo, aqueles formados por gases inertes ou quando ligações $\pi$ atuam como receptores de prótons. ${ }^{98}$ Neste contexto torna-se necessária a execução de cálculos de correlação eletrônica em níveis altamente sofisticados, como os baseados na Teoria de Perturbação de Muitos Corpos (MBPT, Many Body Perturbation Theory) de Møller-Plesset em segunda ordem (MP2), ${ }^{99}$ métodos pós-Hartree-Fock como Interação de Configuração (CI, Configuration Interaction) $)^{100}$ e coupled-cluster CCSD (Coupled Cluster theory limited to Single and Double excitations) $)^{101}$ ou, até mesmo, pela utilização de híbridos da Teoria do Funcional de Densidade (DFT, Density Functional Theory). ${ }^{102-104}$ Através da quantificação das forças de dispersão ou dos termos eletrostáticos, os cálculos teóricos mencionados acima constituem a base para a análise do potencial intermolecular (Equação 3), pelo qual algumas outras propostas de partição de energia também têm sido divulgadas, tais como os códigos DC (Divide and Conquer) de Vaart e Merz Jr. ${ }^{105}$ e a Análise da Decomposição de Energia (EDA, Energy Decomposition Analysis) de $\mathrm{Su}$ e Li. ${ }^{106}$ Entretanto, um dos métodos mais difundidos entre os cientistas teóricos é a Teoria de Perturbação da Simetria Adaptada (SAPT, Symmetry-Adapted Perturbation Theory) desenvolvida por Szalewicz e colaboradores. ${ }^{107,79}$ Em seu algoritmo, a SAPT descreve o dímero A $\cdots$ B como um produto de funções de ordem zero $\left(\Phi_{0}\right)$ para os monômeros isolados $\mathrm{A}\left(\Phi_{\mathrm{A}}\right)$ e $\mathrm{B}\left(\Phi_{\mathrm{B}}\right)$ :

$$
\Phi_{0}=\Phi_{\mathrm{A}} \Phi_{\mathrm{B}}
$$

Como as funções de onda $\Phi_{\mathrm{A}}$ e $\Phi_{\mathrm{B}}$ não se aplicam a um sistema com muitos elétrons, por exemplo a supermolécula intermolecular (A ‥B), utiliza-se o determinante Hartree-Fock com a correlação sendo inserida de maneira perturbativa. De acordo com o método de Møller-Plesset, os Hamiltonianos dos monômeros A e B são desmembrados no formato dos operadores de Fock (F) e de correlação intramonomérica $(\mathrm{W})$ :

$$
\mathrm{W}_{\mathrm{A}}=\mathrm{H}_{\mathrm{A}}-\mathrm{F}_{\mathrm{A}} \text { e } \mathrm{W}_{\mathrm{B}}=\mathrm{H}_{\mathrm{B}}-\mathrm{F}_{\mathrm{B}}
$$

Por sua vez, o operador Hamiltoniano total é composto pelos termos de Fock $\left(\mathrm{F}=\mathrm{F}_{\mathrm{A}}+\mathrm{F}_{\mathrm{B}}\right)$, do potencial de estabilidade intramonomérica ( $\mathrm{W}=\mathrm{W}_{\mathrm{A}}+\mathrm{W}_{\mathrm{B}}$ ) e do operador de interação (núcleos e elétrons) intermolecular não expandido (U): 


$$
\begin{gathered}
\mathrm{H}=\mathrm{F}+\mathrm{U}+\mathrm{W} \\
(\mathrm{F}+\mathrm{U}+\mathrm{W}) \Phi=\mathrm{E} \Phi
\end{gathered}
$$

A Equação 7 representa o formalismo secular de RayleighSchrödinger, ${ }^{108}$ em que a energia de interação SAPT $\left(\mathrm{E}_{\mathrm{Int}}^{\mathrm{SAPT}}\right)$ apresentada na Equação 8 pode ser obtida através da polarização expandida (P) e da repulsão de troca de spin $\left(\mathrm{E}_{\mathrm{x}}\right)$ resultante da função de onda antissimétrica $(\Phi)$ para a troca de elétrons entre as espécies monoméricas. Além disso, os termos de expansão $r, s$ e $t$ são relacionados, respectivamente, ao potencial eletrostático clássico U e às correlações $\mathrm{W}_{\mathrm{A}}$ e $\mathrm{W}_{\mathrm{B}}$, os quais são tratados isoladamente no tunelamento eletrônico de sistemas intermoleculares em estado de interação. ${ }^{81,109-111}$

$$
\mathrm{E}_{\mathrm{Int}}^{\mathrm{SAPT}}=\sum_{r=1}^{\infty} \sum_{s=0}^{\infty} \sum_{t=0}^{\infty}\left(\mathrm{E}_{\mathrm{Pol}}^{r s t}+\mathrm{E}_{\mathrm{Ex}}^{r s t}\right)
$$

Na Equação 9 os componentes SAPT são classificados como descritores de interação para o nível Hartree-Fock ( $\left.\mathrm{E}_{\mathrm{Int}}^{\mathrm{HF}}\right)$ e correlação eletrônica ( $\left.E_{\text {Int }}^{\text {Corr }}\right)$ :

$$
\mathrm{E}_{\mathrm{Int}}^{\mathrm{SAPT}}=\mathrm{E}_{\mathrm{Int}}^{\mathrm{HF}}+\mathrm{E}_{\mathrm{Int}}^{\mathrm{Corr}}
$$

cujos termos $E_{\text {Int }}^{\text {HF }}$ e $E_{\text {Int }}^{\text {Corr }}$ são descritos pelas Equações 10 e 11, respectivamente. Além do termo misto ( $\left.E_{\mathrm{x}}-\mathrm{Ind}\right)$, estas duas equações carregam uma resposta à perturbação Hartree-Fock através dos indicativos Resp, e a energia de interação variacional é contabilizada pelo termo $\delta \mathrm{E}_{\mathrm{HF}, \text { Resp }}$. Em contrapartida, a contribuição $\mathrm{E}^{\mathrm{SCF}-\mathrm{SAPT}}$ que outrora foi denominado como $\mathrm{Mix}^{85}$ conglomera os principais termos $E_{C}, E_{E x}, E_{P o l}$ e $E_{E x-I n d}$ juntamente com a energia Hartree-Fock. Em concordância com Umeyama e Morokuma, ${ }^{85}$ o efeito da correlação eletrônica é contabilizado pelas energias de dispersão, $\mathrm{E}_{\mathrm{D}}^{(20)}+\mathrm{E}_{\mathrm{Ex}-\mathrm{D} \cdot{ }^{(20)}}^{81}$

$$
\begin{aligned}
\mathrm{E}_{\mathrm{Int}}^{\mathrm{HF}}= & \mathrm{E}_{\mathrm{C}}^{(10)}+\mathrm{E}_{\mathrm{Ex}}^{(10)}+\mathrm{E}_{\mathrm{Ind}, \text { Resp }}^{(20)}+\mathrm{E}_{\mathrm{Ex}-\text { Ind, Resp }}^{(20)}+\delta \mathrm{E}_{\mathrm{HF}, \text { Resp }} \\
\mathrm{E}_{\mathrm{Int}}^{\mathrm{Corr}}= & \mathrm{E}_{\mathrm{C}, \text { Resp }}^{(12)}+\mathrm{E}_{\mathrm{Ex}}^{(11)}+\mathrm{E}_{\mathrm{Ex}}^{(12)}+{ }^{\mathrm{t}} \mathrm{E}_{\text {Ind }}^{(22)}+{ }^{\mathrm{t}} \mathrm{E}_{\mathrm{Ex}-\text { Ind }}^{(22)}+ \\
& \mathrm{E}_{\mathrm{D}}^{(20)}+\mathrm{E}_{\mathrm{D}}^{(21)}+\mathrm{E}_{\mathrm{D}}^{(22)}+\mathrm{E}_{\mathrm{Ex}-\mathrm{D}}^{(20)}
\end{aligned}
$$

As características de cada um destes termos são apresentadas na Tabela $1,{ }^{97}$ embora deva ser ressaltado que os aspectos da transferência de carga $\mathrm{E}_{\mathrm{TC}}$ e polarização $\mathrm{E}_{\mathrm{Pol}}$ são incorporados no termo de indução, ${ }^{111}$ e que não há como separá-los devido à energia derivada da transferência de carga ser estritamente dependente dos conjuntos de base. Uma das propostas para obtenção da energia de transferência de carga foi elaborada por Stone e Misquita, ${ }^{112}$ através da diferença das funções de onda tratadas como Bases Centradas nos Complexos (DCBS, Dimer-Centered Basis Sets) e nos Monômeros (MCBS, Monomer-Centered Basis Sets):

$$
\begin{aligned}
\mathrm{E}_{\mathrm{TC}}= & {\left[\mathrm{E}_{\text {Ind, Resp }}^{(20)}+\mathrm{E}_{\mathrm{Ex}-\text { Ind, Resp }}^{(20)}\right] \mathrm{DCB}-} \\
& {\left[\mathrm{E}_{\text {Ind, Resp }}^{(20)}+\mathrm{E}_{\mathrm{Ex} \text {-Ind, Resp }}^{(20)}\right] \mathrm{MCB} }
\end{aligned}
$$

Tabela 1. Termos da partição SAPT utilizados na interpretação de estruturas eletrônicas

\begin{tabular}{lcc}
\hline Termos & Perfil & $\begin{array}{c}\text { Formalismo SAPT utilizado } \\
\text { nos cálculos ab initio }\end{array}$ \\
\hline $\mathrm{E}_{\mathrm{C}}$ & Não cooperativo $^{\mathrm{a}}$ & $\mathrm{E}_{\mathrm{C}}^{(10)}+\mathrm{E}_{\mathrm{C}, \text { Resp }}^{(12)}$ \\
$\mathrm{E}_{\mathrm{Ex}}$ & Cooperativo $^{\mathrm{a}}$ & $\mathrm{E}_{\mathrm{Ex}}^{(10)}+\mathrm{E}_{\mathrm{Ex}}^{(11)}+\mathrm{E}_{\mathrm{Ex}}^{(12)}$ \\
$\mathrm{E}_{\mathrm{Ind}}$ & Cooperativo $^{\mathrm{a}}$ & $\mathrm{E}_{\text {Ind,Resp }}^{(20)}+\mathrm{E}_{\mathrm{Ex}-\text { Ind,Resp }}^{(20)}+\mathrm{C}_{\text {Ind }}^{(22)}+{ }^{\mathrm{t} \mathrm{E}_{\mathrm{Ex}-\text { Ind }}^{(22)}}$ \\
$\mathrm{E}_{\mathrm{D}}$ & Não cooperativo $^{\mathrm{a}}$ & $\mathrm{E}_{\mathrm{D}}^{(20)}+\mathrm{E}_{\mathrm{D}}^{(21)}+\mathrm{E}_{\mathrm{D}}^{(22)}+\mathrm{E}_{\mathrm{Ex}-\mathrm{D}}^{(20)}$ \\
\hline
\end{tabular}

${ }^{a}$ Reproduzida de ref. 97, com permissão da American Chemical Society.
Dependendo do sistema, sua energia pode ser analisada através de uma série SAPTn com n = 0, 2, 2+, que são conhecidos por SAPT0, SAPT2 e SAPT2+, respectivamente: ${ }^{81}$

$$
\begin{aligned}
\mathrm{E}^{\mathrm{SAPT0}=} & \mathrm{E}_{\mathrm{C}}^{(10)}+\mathrm{E}_{\mathrm{Ex}}^{(10)}+\mathrm{E}_{\text {Ind, Resp }}^{(20)}+\mathrm{E}_{\mathrm{Ex}-\text { Ind, Resp }}^{(20)}+ \\
& \mathrm{E}_{\mathrm{D}}^{(20)}+\mathrm{E}_{\mathrm{Ex}-\mathrm{D}}^{(20)}+\delta \mathrm{E}_{\mathrm{HF}, \text { Resp }} \\
\mathrm{E}^{\mathrm{SAPT2} 2}= & \mathrm{E}^{\mathrm{SAPT} 0}+\mathrm{E}_{\mathrm{C}, \text { Resp }}^{(12)}+\mathrm{E}_{\mathrm{C}, \text { Resp }}^{(13)}+\mathrm{E}_{\mathrm{Ex}}^{(11)}+ \\
& \mathrm{E}_{\mathrm{Ex}}^{(12)}+{ }^{\mathrm{t}} \mathrm{E}_{\text {Ind }}^{(22)}+{ }^{\mathrm{t}} \mathrm{E}_{\mathrm{Ex}-\text { Ind }}^{(22)}+\delta \mathrm{E}_{\mathrm{HF}, \text { Resp }} \\
\mathrm{E}^{\mathrm{SAPT2}+}= & \mathrm{E}^{\mathrm{SAPT} 2}+\mathrm{E}_{\mathrm{D}}^{(21)}+\mathrm{E}_{\mathrm{D}}^{(22)}
\end{aligned}
$$

A Equação 13 pode ser resumida para $\mathrm{E}^{\mathrm{SAPTO}}=\mathrm{E}^{\mathrm{SCF}-\mathrm{SAPT}}+$ $\mathrm{E}^{\text {CORR-SAPT }}$, em que os quatro primeiros termos $\left(\mathrm{E}_{\mathrm{C}}^{(10)}, \mathrm{E}_{\mathrm{Ex}}^{(10)}, \mathrm{E}_{\text {Ind,Resp }}^{(10)} \mathrm{e}\right.$ $\mathrm{E}_{\mathrm{Ex}-\mathrm{Ind}, \text { Resp }}^{(20)}$ referentes à Equação 10) comportam a energia do campo autoconsistente (SCF, Self-Consistent Field), ${ }^{113}$ enquanto os dois restantes $\left(\mathrm{E}_{\mathrm{D}}^{(20)} \mathrm{e} \mathrm{E}_{\mathrm{Ex}-\mathrm{D}}^{(20)}\right)$ a dispersão eletrônica. ${ }^{114}$ Determinando a contribuição de cada um destes termos com cálculos realizados em nível $a b$ initio $^{115}$ ou DFT, ${ }^{116-118}$ o método SAPT revela sob quais condições eletrônicas o sistema intermolecular é estabilizado em um mínimo de energia, e com isto torna-se possível designá-lo como forte ou fracamente ligado no que se refere à existência de ligações de hidrogênio ou interações de van der Waals, ${ }^{119}$ respectivamente. Entretanto, é digno de nota que a aplicação do SAPT0 ou SAPT2 requer coerência em relação ao nível de teoria utilizado, ${ }^{120}$ como também o tipo de sistema a ser investigado. Sabe-se que SAPT2 é recomendada para estudos de sistemas grandes contendo elevado grau de efeitos eletrônicos, nos quais o esforço computacional se assemelha à perturbação MP4. ${ }^{121}$

\section{LIGAÇÃO DE HIDROGÊNIO ELETROSTÁTICA}

A análise teórica SAPT tem sido decisiva no estudo de novos sistemas estabilizados através da formação de ligações de hidrogênio. Muito recentemente, Scheiner ${ }^{122}$ apresentou um estudo quântico de ligações de hidrogênio do tipo $\mathrm{X}-\mathrm{Y} \cdots \mathrm{H}-\mathrm{Z} \operatorname{com} \mathrm{X}=\mathrm{H}, \mathrm{Y}=\mathrm{N}$ e Z $=$ $\mathrm{Cl}, \mathrm{HS}, \mathrm{H}_{2} \mathrm{P}$ e $\mathrm{H}_{2} \mathrm{~N}$, e cujos complexos $\mathrm{H}_{3} \mathrm{~N} \cdots \mathrm{HCl}(\mathbf{d}), \mathrm{H}_{3} \mathrm{~N} \cdots \mathrm{SH}_{2}(\mathbf{e})$, $\mathrm{H}_{3} \mathrm{~N} \cdots \mathrm{PH}_{3}(\mathbf{f})$ e $\mathrm{H}_{3} \mathrm{~N} \cdots \mathrm{NH}_{3}(\mathbf{g})$ e suas distribuições de densidades eletrônicas estão ilustrados na Figura 5.

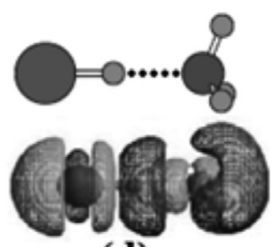

(d)

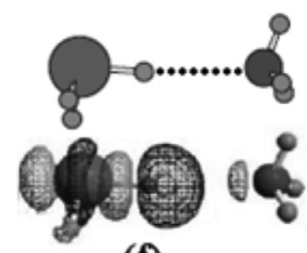

(f)

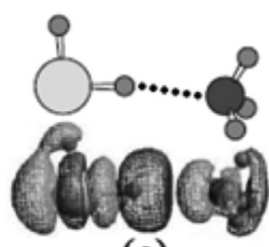

(e)

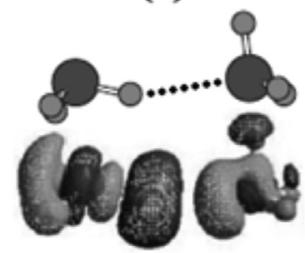

(g)
Figura 5. Geometrias otimizadas dos complexos $\mathrm{H}_{3} \mathrm{~N} \cdots \mathrm{HCl}$ (d), $\mathrm{H}_{3} \mathrm{~N} \cdots \mathrm{HSH}$ (e), $\mathrm{H}_{3} \mathrm{~N} \cdots \mathrm{HPH}_{2}(\boldsymbol{f})$ e $\mathrm{H}_{3} \mathrm{~N} \cdots \mathrm{HNH}_{2}$ (g) obtidas pelo nível de teoria MP2/augcc-pVDZ. Reproduzida da ref. 122, com permissão do American Institute of Physics

Na Tabela 2 são apresentados os resultados da análise SAPT, os valores das energias de interação corrigidas com a remoções do Erro de Superposição do Conjunto de Base (BSSE, Basis Sets 
Superposition Error) ${ }^{123}$ e da Energia vibracional do Ponto Zero (ZPE, Zero-Point Energy). ${ }^{124}$ Através das geometrias otimizadas dos complexos (d), (e), (f) e (g) obtidas a nivel MP2/aug-cc-pVDZ, foram obtidos os respectivos resultados de 1,725; 2,192; 2,629 e 2,281 ̊ para as distâncias intermoleculares acompanhados dos correspondentes valores das energias de interação de $-5,95 ;-1,79$; $+0,04$ e $-1,26 \mathrm{kcal} / \mathrm{mol}$.

Tabela 2. Valores das energias de interação $\Delta \mathrm{E}$ com correções BSSE e $\triangle \mathrm{ZPE}$ obtidos pela aplicação do nível de teoria MP2/aug-cc-pVDZ relacionado à formação dos complexos $\mathrm{H}_{3} \mathrm{~N} \cdots \mathrm{HCl}(\mathbf{d}), \mathrm{H}_{3} \mathrm{~N} \cdots \mathrm{HSH}(\mathbf{e}), \mathrm{H}_{3} \mathrm{~N} \cdots \mathrm{HPH}_{2}$ (f) e $\mathrm{H}_{3} \mathrm{~N} \cdots \mathrm{HNH}_{2}(\mathbf{g})$

\begin{tabular}{|c|c|c|c|c|}
\hline \multirow{2}{*}{ Parâmetros } & \multicolumn{4}{|c|}{ Complexos } \\
\hline & (d) & (e) & (f) & (g) \\
\hline$\Delta \mathrm{E}^{\mathrm{a}}$ & $-9,87$ & $-4,33$ & $-1,53$ & $-3,63$ \\
\hline$(\Delta \mathrm{E}+\mathrm{BSSE})^{\mathrm{a}}$ & $-8,27$ & $-3,28$ & $-0,83$ & $-2,74$ \\
\hline$(\Delta \mathrm{E}+\mathrm{BSSE}+\Delta \mathrm{ZPE})^{\mathrm{a}}$ & $-5,95$ & $-1,79$ & $+0,04$ & $-1,26$ \\
\hline $\mathrm{E}_{\mathrm{C}, \operatorname{Resp}}^{(12)}{ }^{a}$ & $-21,27$ & $-6,95$ & $-1,71$ & $-5,17$ \\
\hline $\mathrm{E}_{\mathrm{Ex}}^{(12) \mathrm{a}}$ & 27,73 & 8,20 & 2,71 & 4,89 \\
\hline${ }^{\prime} \mathrm{E}_{\text {Ind }}^{(22) ~ a}$ & $-14,00$ & $-3,07$ & $-0,87$ & $-1,69$ \\
\hline$\left({ }^{(} \mathrm{E}_{\text {Ind }}^{(22)}+{ }^{t} \mathrm{E}_{\mathrm{Ex}-\operatorname{Ind}}^{(22)}\right)^{\mathrm{a}}$ & $-5,92$ & $-1,32$ & $-0,47$ & $-0,76$ \\
\hline $\mathrm{E}_{\mathrm{D}}^{(20) \mathrm{a}}$ & $-6,23$ & $-2,92$ & $-1,51$ & $-2,04$ \\
\hline $\left.\mathrm{E}_{\mathrm{D}}^{(20)}+\mathrm{E}_{\mathrm{Ex}-\mathrm{D}}^{(20)}\right)^{\mathrm{a}}$ & $-4,82$ & $-2,34$ & $-1,26$ & $-1,67$ \\
\hline
\end{tabular}

Todos os valores são dados em $\mathrm{kcal} / \mathrm{mol}$; ${ }^{a}$ reproduzida da ref. 122 , com permissão do American Institute of Physics.

Observa-se que o sistema $\mathrm{H}_{3} \mathrm{~N} \cdots \mathrm{HCl}$ (d) é o mais fortemente ligado e, por outro lado, o complexo $\mathrm{H}_{3} \mathrm{~N} \cdots \mathrm{HPH}_{2}$ (f) apresenta a interação mais fraca. Pela análise SAPT, a decomposição de energia em função das contribuições $\mathrm{E}_{\mathrm{C}}, \mathrm{E}_{\mathrm{Ex}}, \mathrm{E}_{\mathrm{Pol}}, \mathrm{E}_{\mathrm{D}}$ e $\mathrm{E}_{\mathrm{TC}}$ fornece uma explicação para tal discrepância intermolecular. Analisando isoladamente cada sistema, pode-se observar que a interação mais forte em (d) pode ser explicada pela contribuição eletrostática $\mathrm{E}_{\mathrm{C}, \mathrm{Resp}}^{(20)}$ significativa, fato também comprovado para (e) e (g), embora estes apresentem forças de interação relativamente medianas. Como esperado, a energia proveniente do potencial de troca de spins $\left(\mathrm{E}_{\mathrm{Ex}}^{(12)}\right)$ é sempre positiva, por se tratar de um termo repulsivo derivado do princípio da exclusão de Pauli. Embora o sistema (f) apresente uma contribuição ínfima de $-1,71 \mathrm{kcal} / \mathrm{mol}$ para a energia eletrostática $\mathrm{E}_{\mathrm{C}, \mathrm{Resp}}^{(12)}$, comparativamente os valores de $-1,51 \mathrm{e}-1,26 \mathrm{kcal} / \mathrm{mol} \mathrm{re}-$ lacionados aos termos das energias de dispersão $\mathrm{E}_{\mathrm{D}}^{(20)}$ e $\mathrm{E}_{\mathrm{D}}^{(20)}+\mathrm{E}_{\mathrm{Ex}-\mathrm{D}}^{(20)}$ também se mostraram importantes. De forma incontestável, é sempre recomendado que o estudo teórico de sistemas fracamente ligados, cuja estrutura é estabilizada mediante ação das forças de dispersão, deva ser executado por métodos teóricos eficientes, como o nível MP2/aug-cc-pVDZ. ${ }^{125}$ Nesta mesma linha de investigação, Panek e Jezierska ${ }^{126}$ estudaram ligações de hidrogênio $\mathrm{H}-\mathrm{N} \cdots \mathrm{H}-\mathrm{F}$ em complexos trimoleculares formados pela amônia e o dímero do ácido fluorídrico $\mathrm{NH}_{3} \cdots(\mathrm{HF})_{2}$, como também pelo ácido fluorídrico e o dímero da amônia $\left(\mathrm{NH}_{3}\right)_{2} \cdots \mathrm{HF}$. Os resultados da partição de energia SAPT para estes sistemas são apresentados na Tabela 3.

Duas situações são contempladas, nas quais a funcionalidade ácido(A)-base(B) segue a seguinte ordem: BAA para $\mathrm{NH}_{3} \cdots(\mathrm{HF})_{2}$ ou $\mathrm{NH}_{3} \cdots \mathrm{HF} \cdots \mathrm{HF}$ e BAB para $\left(\mathrm{NH}_{3}\right)_{2} \cdots \mathrm{HF}$ ou $\mathrm{NH}_{3} \cdots \mathrm{HF} \cdots \mathrm{NH}_{3}$. Nestes, analisando a contribuição do dímero $\mathrm{NH}_{3} \cdots \mathrm{HF}$, o termo eletrostático $\mathrm{E}_{\mathrm{C}, \mathrm{Resp}}^{(10)}$ da SAPT0 destaca-se com as contribuições de $-26,95$ e $-23,80 \mathrm{kcal} / \mathrm{mol}$ para a estabilização dos sistemas BAA e BAB, respectivamente. Diante deste domínio da contribuição eletrostática, fica esclarecido o típico portfólio de ligação de
Tabela 3. Resultado da análise SAPT para os complexos $\mathrm{NH}_{3} \cdots(\mathrm{HF})_{2} \mathrm{e}$ $\left(\mathrm{NH}_{3}\right)_{2} \cdots \mathrm{HF}$

\begin{tabular}{|c|c|c|c|}
\hline \multirow{2}{*}{ Partição SAPT } & \multicolumn{3}{|c|}{ Complexos } \\
\hline & $\mathrm{NH}_{3} \cdots \mathrm{HF} \cdots \mathrm{X}_{1}$ & $\mathrm{X}_{2} \cdots \mathrm{HF} \cdots \mathrm{HF}$ & $\mathrm{NH}_{3} \cdots \mathrm{X}_{3} \cdots \mathrm{HF}$ \\
\hline $\mathrm{E}_{\mathrm{C}}^{(10) \mathrm{a}}$ & $-26,95(-23,80)$ & $-8,83(-3,40)$ & $-3,45(-5,58)$ \\
\hline $\mathrm{E}_{\mathrm{Ex}}^{(10) \mathrm{a}}$ & $29,62(24,07)$ & $9,081(1,86)$ & $1,10(5,80)$ \\
\hline $\mathrm{E}_{\text {Ind,Resp }}^{(20)}{ }^{a}$ & $-17,97(-14,73)$ & $-4,58(-0,63)$ & $-0,51(-1,94)$ \\
\hline $\mathrm{E}_{\text {Ex-Ind,Resp }}^{(20)}{ }^{a}$ & $9,29(7,76)$ & $2,42(0,37)$ & $0,28(1,17)$ \\
\hline $\mathrm{E}^{\text {SCF-SAPT a }}$ & $-6,00(-6,70)$ & $-1,92(-1,79)$ & $-2,57(-0,55)$ \\
\hline$\delta \mathrm{E}_{\mathrm{HF}, \text { Resp }}{ }^{\mathrm{a}}$ & $-6,02(-4,72)$ & $-1,22(-0,13)$ & $-0,06(-0,55)$ \\
\hline
\end{tabular}

Todos os valores são dados em $\mathrm{kcal} / \mathrm{mol}$; valores relacionados ao sistema $\mathrm{BAB}$ estão em parênteses; $\mathrm{X}_{1}=\mathrm{X}_{3}=\mathrm{HF}$ e $\mathrm{X}_{2}=\mathrm{NH}_{3}$; ${ }^{\mathrm{a}}$ reproduzida da ref. 126, com permissão da American Chemical Society.

hidrogênio para $\mathrm{H}-\mathrm{N} \cdots \mathrm{H}-\mathrm{F}$, embora $\mathrm{H}-\mathrm{F} \cdot \mathrm{H}-\mathrm{H}-\mathrm{F}$ seja formada com contribuições de $\mathrm{E}_{\mathrm{C}, \mathrm{Resp}}^{(10)}$ mais amenas, cujos valores são -8,83 (BAA) e $-3,40 \mathrm{kcal} / \mathrm{mol}$ (BAB).

Os resultados apresentados acima são abordagens parciais, pois a estrutura trimérica real é desconsiderada. Como bem estabelecido, complexos trimoleculares têm sua estabilidade avaliada pelas energias decorrentes de duas interações existentes $(\mathrm{H}-\mathrm{N} \cdots \mathrm{H}-\mathrm{F}$ e H-F $\cdots \mathrm{H}-\mathrm{F})$, para as quais foram obtidos os valores de $-20,71$ e $-17,85 \mathrm{kcal} / \mathrm{mol}$ nos sistemas $\mathrm{NH}_{3} \cdots(\mathrm{HF})_{2}$ e $\left(\mathrm{NH}_{3}\right)_{2} \cdots \mathrm{HF},{ }^{126}$ respectivamente. Estes resultados corroboram satisfatoriamente os valores de -22,75 e -19,21 $\mathrm{kcal} / \mathrm{mol}$ calculados pela SAPT. Todavia, deve ser mencionado que variações drásticas de polarização são características de estruturas multiconectadas intermolecularmente, e que os valores de -17,97 e $-14,73 \mathrm{kcal} / \mathrm{mol}$ são considerados parcelas importantes na formação das ligações de hidrogênio $\mathrm{H}-\mathrm{N} \cdots \mathrm{H}-\mathrm{F}$ em BAA e BAB, respectivamente. Desta forma, através da energia de indução (polarização e transferência de carga) pode ser assegurada a existência do efeito energético cooperativo característico de clusters moleculares, ${ }^{127,128}$ conforme já demonstrado nos trabalhos de Sánches et al., Grabowski e Bilewicz, ${ }^{129}$ Canuto et al.,${ }^{130}$ Frontera et al.,${ }^{131}$ Parra et al. ${ }^{132} \mathrm{e}$ em recente comunicação de Bauer e Spange. ${ }^{133}$

\section{INTERAÇÃO DE van der WAALS DISPERSIVA}

Em um artigo de revisão, Hobza e Zahradník ${ }^{134}$ apresentaram o seguinte argumento: "Sistemas van der Waals podem ser formados por qualquer tipo de molécula, íon ou radical". Esta asseveração procede pelo fato das forças de dispersão estarem presentes em toda natureza. Porém, um universo tão abrangente como este impõe algumas particularidades como, por exemplo, a dificuldade em captar a fraca intensidade destas forças, visto que atuam em sistemas com grande separação intermolecular e fraca energia de dissociação. ${ }^{135}$ Quando formado unicamente por moléculas, o sistema resultante é estabilizado por uma interação de van der Waals, a qual inegavelmente ocorre com mais frequência entre gases inertes, ${ }^{136}$ como observado na formação de dímeros de $\operatorname{argônio}{ }^{137}$ e sistemas mistos com kriptônio. ${ }^{138} \mathrm{Na}$ restrita química reacional destes gases, Räsänen et al. ${ }^{139}$ registraram a existência do primeiro composto formado em baixas temperaturas por elementos ligados ao argônio, o fluoridreto de argônio (HArF) na forma de par iônico com uma ligação covalente H-Ar e outra iônica $(\mathrm{HAr})^{+}-\mathrm{F}^{-}$. Nesta última, o íon fluoreto assemelha-se ao $\mathrm{Y}^{-\delta}$ do modelo da ligação de hidrogênio $\left(\mathrm{X}-\mathrm{Y}^{-\delta} \cdots \mathrm{H}^{+\delta}-\mathrm{Z}\right) \mathrm{e}$, assim, a possibilidade do composto HArF interagir intermolecularmente mostrou-se plausível. Foi a partir desta ideia que Cukras e Sadlej ${ }^{140}$ discutiram a formação dos complexos lineares HArF $\cdots \mathrm{N}_{2}$ e HArF $\cdots \mathrm{P}_{2}$. Contrariamente à intuição revisitada acima, os sistemas estudados apresentaram HArF 
como o doador de próton formando duas interações $\mathrm{H} \cdots \mathrm{N}(\mathbf{h})$ e $\mathrm{H} \cdots \mathrm{P}(\mathbf{j})$, embora suas estruturas assimétricas permitam uma polaridade a ponto do argônio compartilhar a mesma região eletropositiva do hidrogênio. Este aspecto projeta a molécula de nitrogênio a interagir

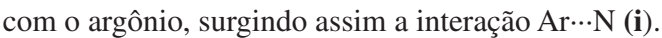

Os resultados da análise SAPT para os complexos (h), (i) e (j) estão organizados na Tabela 4 e ilustrados na Figura 6.

Tabela 4. Resultado da análise SAPT para os complexos HArF $\cdots \mathrm{N}_{2}$ e HArF $\cdots \mathrm{P}_{2}$

\begin{tabular}{|c|c|c|c|}
\hline \multirow{2}{*}{ Partição SAPT } & \multicolumn{3}{|c|}{ Complexos } \\
\hline & $\mathrm{HArF} \cdots \mathrm{N}_{2}(\mathbf{h})$ & $\mathrm{HArF} \cdots \mathrm{N}_{2}(\mathbf{i})$ & $\operatorname{HArF} \cdots \mathrm{P}_{2}(\mathbf{j})$ \\
\hline $\mathrm{E}_{\mathrm{C}}^{(10) \mathrm{a}}$ & $-16,49$ & $-8,65$ & $-12,09$ \\
\hline $\mathrm{E}_{\mathrm{C}, \mathrm{Resp}}^{(12)}{ }^{\mathrm{a}}$ & $-3,91$ & $-2,38$ & $-7,83$ \\
\hline $\mathrm{E}_{\mathrm{C}}^{\mathrm{a}}$ & $-20,39$ & $-11,03$ & $-19,92$ \\
\hline $\mathrm{E}_{\mathrm{Ex}}^{(10) \mathrm{a}}$ & 26,58 & 11,74 & 56,48 \\
\hline $\mathrm{E}_{\mathrm{Ex}}^{(11) \mathrm{a}}$ & 1,10 & 0,06 & 3,95 \\
\hline $\mathrm{E}_{\mathrm{Ex}}^{(12) \mathrm{a}}$ & 5,41 & 2,62 & 3,69 \\
\hline $\mathrm{E}_{\mathrm{Ex}}^{\mathrm{a}}$ & 33,10 & 14,43 & 64,22 \\
\hline $\mathrm{E}_{\text {Ind,Resp }}^{(20)}{ }^{a}$ & $-16,25$ & $-6,94$ & $-56,20$ \\
\hline $\mathrm{E}_{\mathrm{Ex}, \mathrm{Resp}}^{(20)}{ }^{\mathrm{a}}$ & 7,70 & 4,27 & 29,96 \\
\hline $\mathrm{t}_{\text {Ind }}^{(22) \mathrm{a}}$ & $-1,47$ & $-1,10$ & $-3,32$ \\
\hline${ }^{\prime} \mathrm{E}_{\mathrm{Ex}-\mathrm{Ind}}^{(22)}{ }^{\mathrm{a}}$ & 0,69 & 0,68 & 1,89 \\
\hline $\mathrm{E}_{\text {ind }} \mathrm{a}^{\mathrm{a}}$ & $-9,33$ & $-3,08$ & $-24,07$ \\
\hline $\mathrm{E}_{\mathrm{D}}^{(20) \mathrm{a}}$ & $-11,64$ & $-9,95$ & $-23,70$ \\
\hline $\mathrm{E}_{\mathrm{Ex}-\mathrm{D}}^{(20)} \mathrm{a}^{\mathrm{a}}$ & 1,61 & 1,14 & 3,24 \\
\hline $\mathrm{E}_{\mathrm{D}}{ }^{\mathrm{a}}$ & $-10,03$ & $-8,81$ & $-20,46$ \\
\hline $\mathrm{dE}_{\mathrm{HF}, \operatorname{Resp}}^{\mathrm{a}}$ & $-6,08$ & $-0,71$ & $-19,50$ \\
\hline $\mathrm{E}^{\mathrm{SAPTO} a \mathrm{a}}$ & $-8,49$ & $-8,38$ & 1,29 \\
\hline $\mathrm{E}^{\mathrm{SAPT2} \text { a }}$ & $-18,70$ & $-9,21$ & $-19,84$ \\
\hline
\end{tabular}

Todos os valores são dados em $\mathrm{kcal} / \mathrm{mol}$; ${ }^{a}$ reproduzida da ref. 140 , com permissão da Elsevier.

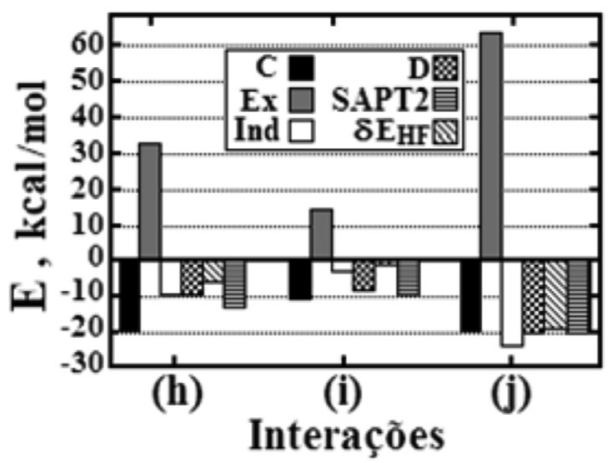

Figura 6. Decomposição SAPT para os sistemas $\mathrm{HArF} \cdots \mathrm{N}_{2}$ (h e i $)$ e $\mathrm{HArF} \cdots \mathrm{P}_{2}$ (j). Reproduzida da ref. 140, com permissão da Elsevier

Inicialmente, constata-se que a energia eletrostática $\mathrm{E}_{\mathrm{C}}$ é dominante, devido aos elevados valores de -20,39; -11,03 e -19,92 kcal/mol obtidos para (h), (i) e (j), respectivamente. Estas energias altamente atrativas são suprimidas pelas energias de repulsão de troca $\mathrm{E}_{\mathrm{Ex}}$, cujos valores correspondentes são 33,10; 14,43 e 64,11 kcal/mol. Como já citado, a indução obtida pela SAPT inclui os fenômenos de polarização e transferência de carga e, pelos resultados diagramatizados na Figura 6, verifica-se que a contribuição $\mathrm{E}_{\text {Ind }} \mathrm{de}-3,08 \mathrm{kcal} / \mathrm{mol}$ é mínima para a estabilização do complexo (i), mas em contrapartida o seu valor de $-24,07 \mathrm{kcal} / \mathrm{mol}$ em (j) é mais atrativo do que a contribuição eletrostática $\mathrm{E}_{\mathrm{C}}$. Nesta situação aparentemente degenerada não haveria tendência de estabilização mas, a energia de dispersão fornece uma contribuição atrativa substancial. Isto é verificado em (j), cujo valor de -20,46 kcal/mol para $\mathrm{E}_{\mathrm{D}}$ é ligeiramente mais estável do que $\mathrm{E}_{\mathrm{C}}$ de $-19,92 \mathrm{kcal} / \mathrm{mol}$. Cukras e Sadlej ${ }^{140}$ admitiram que a estabilização de sistemas fracamente ligados poderia ser discutida pela razão entre os efeitos de indução (polarização acentuada e transferência de carga) e dispersão da seguinte maneira: $\mathrm{E}_{\text {Ind,Resp }}^{(20)} / \mathrm{E}_{\mathrm{D}}^{(20)}$. Por esta relação, os resultados de 1,4 para (h), 0,7 para (i) e 2,3 para (j) mostram que a indução e a dispersão se revelam equivalentes, ${ }^{141}$ exceto para (i) mas, no geral, observa-se uma leve proeminência indutiva. Esta não é uma questão puramente interpretativa, uma vez que o fator geométrico pode influenciar decisivamente na contribuição de cada termo da SAPT, conforme mostrado nos trabalhos de Jaeger et al., Tarakeshwar e Kim. ${ }^{142}$

Apesar deste cenário reportado por Cukras e Sadlej ${ }^{140}$ apontar uma importância aparentemente secundária das forças de dispersão, Panek et al. ${ }^{138}$ mostraram que estas forças (valores médios de $-1 \mathrm{kcal} / \mathrm{mol}$ ) regem a formação de complexos formados por argônio, kriptônio e ácido fórmico $\left(\mathrm{CH}_{2} \mathrm{O}_{2} \cdots \mathrm{Ar}\right.$ e $\left.\mathrm{CH}_{2} \mathrm{O}_{2} \cdots \mathrm{Kr}\right)$ como sendo estabilizados por interações de van der Waals, e que a contribuição eletrostática é praticamente inexistente. Por outro lado, o complexo formado através da ligação de hidrogênio $\mathrm{O} \cdots \mathrm{H}$ entre água e ácido fórmico $\left(\mathrm{CH}_{2} \mathrm{O}_{2} \cdots \mathrm{H}_{2} \mathrm{O}\right)$ é estabilizado por forças puramente elestrostáticas $\left(\mathrm{E}_{\mathrm{C}}^{(10)}\right)$ em uma razão 3:1 em relação às forças dispersivas. É natural que, independente do sistema fracamente ligado formado por gases inertes ser considerado o diferencial em comparação com os complexos de hidrogênio, a diferença energética entre eles está na relação eletrostática/dispersiva. Como já observado em outro trabalho de Cybulski e Sadlej, ${ }^{143}$ esta razão discrimina sistemas intermoleculares com energias de interação bastante distintas que, além dos complexos de gases inertes, também mostra que as interações stacking apresentam destaque para a atuação das forças dispersivas, ${ }^{99,144}$ que por serem diminutas provocam flutuações de carga com indução instântanea de dipolos. ${ }^{145}$ Tradicionalmente, as forças de dispersão características de sistemas fracamente ligados têm sido consideradas fator limitante para alguns métodos teóricos, em particular os funcionais híbridos da DFT. Para isso foi desenvolvido o método SAPT(DFT) ${ }^{146} \mathrm{e}$, de acordo com Podeszwa e Szalewicz, ${ }^{147}$ este algoritmo descreve satisfatoriamente sistemas formados por gases inertes e benzeno. Diante do exposto, a SAPT supera o critério subjetivo da força de interação e, de fato, foi neste cenário que Riley e Hobza ${ }^{111}$ conseguiram diferenciar algumas interações de halogênio consideradas semelhantes ( $\mathrm{I} \cdots \mathrm{O}, \mathrm{Cl} \cdots \mathrm{O}$, e $\mathrm{Br} \cdots \mathrm{O}$ ), em que a contribuição eletrostática domina a estabilização do sistema $\mathrm{H}_{3} \mathrm{CI} \cdots \mathrm{OCH}_{2}$, enquanto a dispersão é a responsáveil pela formação dos complexos $\mathrm{H}_{3} \mathrm{CCl} \cdots \mathrm{OCH}_{2}$ e $\mathrm{H}_{3} \mathrm{CBr} \cdots \mathrm{OCH}_{2}$.

\section{IMPACTO ESPECTROSCÓPICO}

A força de interação de sistemas formados por ligação de hidrogênio ou interação de van der Waals atribui, além da estabilização intermolecular, um impacto espectroscópico na ligação $\mathrm{H}-\mathrm{Z}$ devido à variação na sua frequência de estiramento e, em alguns casos, aumento na intensidade de absorção na região do infravermelho. ${ }^{148}$ Esta variação pode ser compreendida de duas maneiras: ${ }^{13}$ efeito batocrômico ou red-shift - descolamento da frequência para valores mais baixos no espectro de absorção ${ }^{149}$ e, efeito hipsocrômico ou blue-shift - descolamento da frequência para valores mais altos no espectro de absorção. ${ }^{150} \mathrm{~A}$ literatura especializada mostra que efeitos red-shifts são observados em sistemas intermoleculares fortemente ligados, enquanto complexos que interagem de forma mais branda apresentam os eventos blue-shifts. ${ }^{151} \mathrm{Em}$ outras palavras, sistemas drasticamente perturbados pela intensa força de interação provocam 
o surgimento dos efeitos red-shifts, enquanto os blue-shifts são originados em complexos que praticamente mantêm a integridade estrutural devido à fraca interação intermolecular. ${ }^{152}$ Encontrar uma conexão entre os deslocamentos químicos red-shifts e blue-shifts e a força de interação sempre foi pertinente, pois haveria a possibilidade de relacioná-los à formação de ligações de hidrogênio e interações de van der Waals, respectivamente.

Analisando a origem física da ligação de hidrogênio, Hermansson ${ }^{153}$ admitiu que a região de alta densidade eletrônica $X-\mathrm{Y}^{-\delta}$ poderia gerar um campo elétrico uniforme na espécie doadora de próton $\mathrm{H}^{+\delta}-\mathrm{Z}$. Este fenômeno de indução provoca uma alteração no momento de dipolo em função do comprimento da ligação química $\left(\mu=q \cdot r_{H Z}\right)$ do doador de próton quando isolado $\left(\mu^{\circ}\right)$ e quando ocorre a formação do complexo intermolecular $\left(\mu^{\text {Ind }}\right)$. Dependendo da intensidade do campo elétrico gerado por $\mathrm{X}-\mathrm{Y}^{-\delta}$, a indução no momento de dipolo influi no deslocamento da frequência $\left(\Delta v^{\text {Str }}\right)$ de estiramento de $\mathrm{H}^{+\delta}-\mathrm{Z}$ conforme a Equação 16:

$$
\Delta v^{S t r}=-f\left(\frac{d \mu^{\mathrm{o}}}{d r_{H Z}}+\frac{1}{2} \frac{d \mu^{I n d}}{d r_{H Z}}\right)
$$

O efeito red-shift é interpretado pelo aumento no comprimento da ligação $\mathrm{r}_{\mathrm{Hz}}$ ocasionado pelo aumento do momento dipolar induzido $\mu^{\text {Ind }}$. Isto ocorre apenas quando há influência de um campo elétrico forte ou alta energia de interação e na Equação 16 a relação entre $\mu^{\text {Ind }}$ e $r_{H z}$ torna a segunda fração positiva, resultando em um

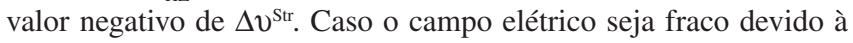
baixa energia de interação, a segunda fração é negativa e o resultado para $\Delta v^{\text {Str }}$ é positivo, o que caracteriza o efeito blue-shift. Embora Nesbitt ${ }^{154}$ tenha destacado que sistemas intermoleculares essencialmente dispersivos formados por gases inertes, tais como $\mathrm{Ne} \cdots \mathrm{HCl}$, $\mathrm{Ar} \cdots \mathrm{HCl}$ e $\mathrm{Ar} \cdots \mathrm{N}_{2} \mathrm{O}$, tenham o efeito blue-shift como sua principal característica espectroscópica, alguns trabalhos têm mostrado que a origem dos efeitos blue-shift provém das forças de dispersão, ${ }^{155} \mathrm{da}$ transferência de carga inerente à energia de indução ${ }^{156}$ ou da energia eletrostática. ${ }^{89,157}$ De acordo com Cukras e Sadlej, ${ }^{140}$ a indução também pode provocar efeitos red-shifts em sistemas fracamente ligados e, portanto, nenhuma correlação efetiva pode ser obtida entre a Equação 16 e os resultados fornecidos pela SAPT. ${ }^{158}$

\section{O LIMITE DA FORÇA DE INTERAÇÃO: COVALÊNCIA}

Em todos esses anos, detectar redundância covalente em sistemas intermoleculares sempre foi considerada uma descoberta inovadora. ${ }^{159}$ Em geral, sabe-se que o caráter covalente de uma interação intermolecular gira em torno de $10 \%$ e que os $90 \%$ restantes consistem em um estado considerado puramente eletrostático. ${ }^{10} \mathrm{Em}$ um recente artigo de revisão intitulado What Is the Covalency of Hydrogen Bonding?, Grabowski ${ }^{36}$ revisita todos os aspectos teóricos relacionados à condição covalente da ligação de hidrogênio, destacando principalmente a densidade eletrônica intermolecular. Além da DFT ${ }^{160} \mathrm{e}$ da teoria de Hirsfield, ${ }^{161}$ a Teoria Quântica de Átomos em Moléculas (QTAIM, Quantum Theory of Atoms in Molecules) ${ }^{162}$ desenvolvida por Bader também adota a densidade eletrônica como observável mecânico-quântica para descrição de carga e momento dipolar em sistemas de camada aberta com contribuição cooperativa de suas subunidades, as quais se entrelaçam em uma linha de pensamento chamada de “... trajetória de Dalton à Schrödinger". ${ }^{163}$ Em consoante com recentes revisões, ${ }^{91,164}$ é sempre destacado que a QTAIM modela o sistema químico como sendo constituído por uma superfície $S(\Omega, r)$ com fluxo nulo de carga eletrônica:

$$
\nabla \rho_{(\mathrm{r})} \cdot \mathrm{n}_{(\mathrm{r})}=0 \quad \forall(\mathrm{r}) \in \mathrm{S}_{(\Omega, \mathrm{r})}
$$

Governada pelo princípio da ação estacionária de Schwinger, ${ }^{165} \mathrm{a}$ cooperatividade eletrônica é tratada por um deslocamento de carga e que as contribuições das subunidades são contabilizadas através de uma força $(\mathrm{F})$ exercida pelos elétrons distribuídos de forma média:

$$
F_{(\Omega)}=-\oint d S_{(\Omega, r)} \vec{\sigma}_{(r)} n_{(r)}
$$

onde $\vec{\sigma}_{(\mathrm{r})}$ é o fluxo de densidade do momento de deslocamento eletrônico. Tratada de uma forma local, a Equação 18 gera o teorema virial da densidade eletrônica em função das energias cinética $(\mathrm{G})$ e potencial (U) da densidade eletrônica local:

$$
\left(\frac{\hbar^{2}}{4 m}\right) \nabla^{2} \rho=2 G+U
$$

Cremer e Kraka ${ }^{166}$ utilizaram esta expressão para avaliar o máximo de densidade eletrônica capaz de distinguir ligações covalentes e interações intermoleculares (ligações de hidrogênio e interações de van der Waals), em que foi proposta a definição de energia de densidade eletrônica $\mathrm{H}:{ }^{167}$

$$
\mathrm{H}=\mathrm{G}+\mathrm{U}
$$

Como G e U são energias positivas e negativas, a energia da densidade eletrônica pode ser interpretada como: $\mathrm{H}<0$ (estabilidade com acúmulo de densidade eletrônica) ou $\mathrm{H}>0$ (instabilidade com depressão de densidade eletrônica). Grabowski et al. ${ }^{168}$ relacionaram estes argumentos com a partição SAPT, admitindo $\mathrm{E}_{\mathrm{Ind}}=\mathrm{E}_{\mathrm{Pol}}+\mathrm{E}_{\mathrm{TC}} \mathrm{e}$ $\mathrm{E}_{\mathrm{D}}=\mathrm{E}_{\text {Corr }}$, o que torna a Equação 3 equivalente à 21:

$$
\mathrm{U}=\mathrm{E}_{\mathrm{C}}+\mathrm{E}_{\mathrm{Ex}}+\mathrm{E}_{\text {Ind }}+\mathrm{E}_{\mathrm{Corr}}
$$

Sabe-se que altos índices de covalência estão associados com elevada indução devido à transferência de carga, a qual é drasticamente afetada pelo BSSE, que é corrigido pelo método counterpoise de Boys e Bernardi. ${ }^{123}$ De forma análoga a Cukras e Sadlej j ${ }^{140}$ que utilizaram a relação $\mathrm{E}_{\text {Ind,Resp }}^{(20)} / \mathrm{E}_{\mathrm{D}}^{(20)}$ para avaliar a estabilização de sistemas fracamente ligados, Grabowski et al. ${ }^{168}$ consideraram a contribuição eletrostática $\mathrm{E}_{\text {Ind,Resp }}^{(20)} / \mathrm{E}_{\mathrm{C}, \text { Resp }}^{(12)}$ para medir o caráter covalente das ligações químicas em função da distância interatômica, gerando um diagrama ilustrado na Figura 7. Percebe-se claramente que valores de $\mathrm{E}_{\text {Ind,Resp }}^{(20)} / \mathrm{E}_{\mathrm{C}, \mathrm{Resp}}^{(12)}$ superiores à fixa de 0,45 relacionados com comprimento de ligação menores que 1,2 ̊̊ indicam que Pol e TC fornecem uma parcela maciça para a estabilização do sistema químico covalente como, por exemplo, o íon (HFH) ${ }^{-}$. Em outras palavras, nesta circunstância há uma elevada concentração de carga observando-se, em termos da aproximação QTAIM, um valor negativo do Laplaciano da densidade eletrônica $\nabla^{2} \rho$, que por sua vez contribui para $\mathrm{H}<0$. Para distâncias de interação maiores que 1,6 $\AA$ observa-se uma tendência de diminuição das contribuições Pol e TC, em que os sistemas intermoleculares $\mathrm{CO}_{2} \mathrm{H}_{2} \cdots \mathrm{CO}_{2} \mathrm{H}_{2}$ e $\mathrm{H}_{2} \mathrm{O} \cdots \mathrm{H}_{2} \mathrm{O}$ apresentam caráter covalente parcial e nulo, respectivamente, mas os perfis de suas energias de densidade eletrônica $\mathrm{H}$ podem ser negativos (estável e parcialmente covalente) ou positivos (instável e não covalente) com destaque para $\nabla^{2} \rho>0$. Em corroboração com Rozas e colaboradores, ${ }^{169}$ sistemas intermoleculares formados por ligação de hidrogênio ou interação de van der Waals apresentam $\nabla^{2} \rho>0$, além dos valores de densidade eletrônica serem menores do que $0,003 \mathrm{e} /{ }_{\text {ao }} \cdot 3,16,17$

Na Tabela 5 são apresentados os principais critérios utilizados para o estudo de sistemas intermoleculares. Como já discutido, a força de interação é considerada um critério subjetivo e interpretativo e sistemas formados via ligação de hidrogênio podem apresentar efeitos vibracionais red-shift ou blue-shift, independentemente das 


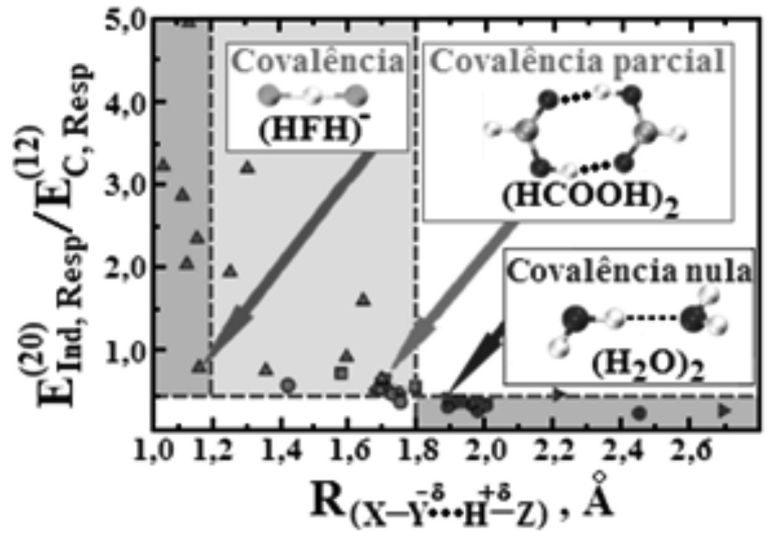

Figura 7. Classificação da covalência em interações intermoleculares através

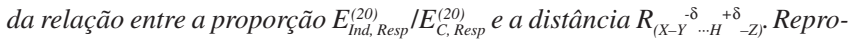
duzida da ref. 36, com permissão da American Chemical Society

Tabela 5. Parâmetros que distinguem ligação de hidrogênio e interação de van der Waals

\begin{tabular}{|c|c|c|}
\hline \multirow[b]{2}{*}{ Parâmetros } & \multicolumn{2}{|c|}{ Características } \\
\hline & $\begin{array}{l}\text { Ligação de } \\
\text { hidrogênio }\end{array}$ & $\begin{array}{c}\text { Interação de } \\
\text { van der Waals }\end{array}$ \\
\hline Força de interação $(\Delta \mathrm{E})^{\mathrm{a}}$ & forte $(12-24)^{e}$ & fraca $(<12)^{\mathrm{e}}$ \\
\hline Natureza física $(\mathrm{SAPT})^{\mathrm{b}}$ & eletrostática & dispersiva \\
\hline $\begin{array}{l}\text { Efeito vibracional } \\
\left(\Delta v_{\mathrm{H}}{ }^{+\delta}{ }_{-\mathrm{Z}}\right)^{\mathrm{c}}\end{array}$ & red-shift ou blue-shift & red-shift ou blue-shift \\
\hline Covalência (QTAIM) ${ }^{\mathrm{d}}$ & $\mathrm{H}>0$ & $\mathrm{H}>0$ \\
\hline
\end{tabular}

$\Delta \mathrm{E} \mathrm{em} \mathrm{kcal} / \mathrm{mol}$; ${ }^{a}$ refs. 78,167 ; ${ }^{b}$ ref. 158 ; ${ }^{c}$ ref. 154 ; ${ }^{d}$ refs. $36,167,168$; ${ }^{\text {refs. }}$ 170,171 .

suas naturezas físicas. A aplicação da QTAIM mostra-se capaz de diferenciar ligações químicas covalentes das interações intermoleculares, mas esta análise se mostra limitada a certo nível, pois a energia da densidade eletrônica $\mathrm{H}$ apresenta um único perfil $(\mathrm{H}>0)$ se o sistema intermolecular é formado por ligação de hidrogênio ou interação de van der Waals, conforme mostrado em um recente comentário de Szatyłowicz. ${ }^{170}$ Entretanto, esta força de interação está sujeita à natureza da composição eletrônica do sistema intermolecular, que pode ser formado mediante ação coulômbica ou dispersiva. Estas duas realidades são os únicos parâmetros que justificam a formação das ligações de hidrogênio e interações de van der Waals, e é neste sentido que a metodologia SAPT se mostra totalmente eficaz.

\section{CONCLUSÃO}

Nesta revisão foram discutidos os principais tipos de forças existentes na natureza e como os sistemas químicos são dependentes da essência física de cada uma delas, que podem ser resumidas nas energias eletrostáticas, troca de spin, polarização, transferência de carga e dispersão. É vastamente conhecido que a atuação distinta destas energias contribui para a imensa diversidade química mas, em se tratanto de sistemas intermoleculares, estes são formados em sua maioria por ligações de hidrogênio eletrostáticas. Todavia, ainda persiste a dúvida se ligações de hidrogênio podem ser tratadas como interações de van der Waals. As ligações de hidrogênio com energias superiores a $5 \mathrm{kcal} / \mathrm{mol}^{171,172}$ não podem ser constituídas unicamente por forças de dispersão, mas principalmente por atração eletrostática. A interface entre estes dois eventos necessita ser conhecida, para que o sistema intermolecular possa ser caracterizado através de ligações de hidrogênio ou interações de van der Waals. Neste nível de análise, apenas a decomposição de energia SAPT pode fornecer o quinhão dispersivo e/ou eletrostático sendo, portanto, um critério essencial nos estudos dos mais variados tipos de sistemas intermoleculares. ${ }^{173}$

\section{AGRADECIMENTOS}

FAPESB, CNPq e CAPES.

\section{REFERÊNCIAS}

1. Alberto, S. A. A.; Rev. USP 2005, 66, 14; Brumfiel, G.; Nature 2011, 479, 456.

2. Silva, P. R.; Rev. Bras. Ensino Fís. 2008, 30, 3304.

3. Fortes, E. C. F. S.; Tijero, M. C.; Pleitez, V.; Rev. Bras. Ensino Fís. 2007, 29, 415 .

4. da Silva, C. O.; Natti, P. L; Rev. Bras. Ensino Fís. 2007, 29, 175.

5. Wales, D. J.; Intermolecular Forces and Clusters II, Springer: Berlin, 2005.

6. Rzepa, H. S.; Nat. Chem. 2009, 1, 510; Rabinovich, D.; J. Chem. Educ. 2003, 80, 31 .

7. Nascimento, M. A. C.; J. Braz. Chem. Soc. 2008, 19, 245.

8. Alkorta, I.; Rozas, I.; Elguero, J.; Chem. Rev. Soc. 1998, 27, 163.

9. Biswal, H. S.; Wategaonkar, S.; J. Phys. Chem. A 2009, 113, 12763.

10. Martin, T. W.; Derewenda, Z. S.; Nature 1999, 6, 403.

11. Volkov, A.; King, H. F.; Coppens, P.; J. Chem. Theor. Comput. 2006, 2, 81 .

12. Oliveira, B. G.; Araújo, R. C. M. U.; Carvalho, A. B.; Ramos, M. N.; J. Mol. Model. 2009, 15, 123; Remer, L. C.; Jensen, J. H.; J. Phys. Chem. A 2000, 104, 9266.

13. Oliveira, B. G.; Lima, M. C. A.; Pitta, I. R.; Galdino, S. L.; Hernandes, M. Z.; J. Mol. Model. 2010, 16, 119; Olovsson, I.; Z. Phys. Chem. 2006, 220, 963.

14. Oliveira, B. G.; Araújo, R. C. M. U.; Carvalho, A. B.; Ramos, M. N.; Struct. Chem. 2009, 20, 663; Oliveira, B. G.; Araújo, R. C. M. U.; Carvalho, A. B.; Ramos, M. N.; Quim. Nova 2007, 30, 1167; Scheiner, S.; Annu. Rev. Phys. Chern. 1994, 45, 23.

15. Oliveira, B. G.; Araújo, R. C. M. U.; Ramos, M. N.; J. Mol. Struct. (THEOCHEM) 2009, 908, 79; Nishio, M.; Tetrahedron 2005, 61, 6923.

16. Oliveira, B. G.; Araújo, R. C. M. U.; Pereira, F. S.; Lima, E. F.; Silva, W. L. V.; Carvalho, A. B.; Ramos, M. N.; Quim. Nova 2008, 31, 1673.

17. Oliveira, B. G.; Araújo, R. C. M. U.; Monatsh. Chem. 2011, 142, 861.

18. Oliveira, B. G.; Vasconcellos, M. L. A. A.; Inorg. Chem. Commun. 2009, 12, 1142 .

19. Oliveira, B. G.; Vasconcellos, M. L. A. A.; Struct. Chem. 2009, $20,897$.

20. Oliveira, B. G.; Vasconcellos, M. L. A. A.; Olinda, R. R.; Alencar Filho, E. B.; Struct. Chem. 2009, 20, 81; Jabłoński, M.; Palusiak, M.; J. Phys. Chem. A 2012, 116, 2322.

21. Oliveira, B. G.; Araújo, R. C. M. U.; Ramos, M. N.; Struct. Chem. 2008, 19, 665; Oliveira, B. G.; Araújo, R. C. M. U.; Ramos, M. N.; Struct. Chem. 2008, 19, 185; Oliveira, B. G.; Araújo, R. C. M. U.; Silva, J. J.; Ramos, M. N.; Struct. Chem. 2010, 21, 221; Giambiagi, M. M. S.; Bultinck, P.; J. Braz. Chem. Soc. 2008, 19, 263.

22. Lewis, G. N.; Valence and the Structure of Atoms and Molecules, Chemical Catalog Co. Reprinted: New York, 1923; Jensen, W. B; Chem. Rev. 1978, 78, 1; Costa, P. R. R.; Ferreira, V. F.; Esteves, P. M.; Vasconcellos, M. L. A. A.; Ácidos e Bases em Química Orgânica, Bookman: Porto Alegre, 2005.

23. Araújo, R. C. M. U.; Ramos, M. N.; J. Braz. Chem. Soc. 1998, 9, 499; Parnaíba- Silva, A. J.; Silva, J. B. P.; Gama, A. A. S.; Ramos, M. N.; J. Braz. Chem. Soc. 2006, 17, 237. 
24. Bueno, W. A.; Quim. Nova 1992, 15, 328; Bartha, F.; Kapuy, O.; Kozmutza, C.; van Alsenoy, C.; J. Mol. Struct. (THEOCHEM) 2003, 666-667, 117; Ludwig, R.; Phys. Chem. Chem. Phys. 2002, 4, 5481; Luck, W. A. P.; J. Mol. Struct. 1998, 448, 131; Gilli, G.; Gilli, P.; J. Mol. Struct. 2000, 552, 1; http://www.ggilli.com, acessada em Junho 2012; http://www.hbond.de, acessada em Maio 2012.

25. Arunan, E.; Desiraju, G. R.; Klein, R. A.; Sadlej, J.; Scheiner, S.; Alkorta, I.; Clary, D. C.; Crabtree, R. H.; Dannenberg, J. J.; Hobza, P.; Kjaergaard, H. G.; Legon, A. C.; Mennucci, B.; Nesbitt, D. J.; Pure Appl. Chem. 2011, 83, 1619; Arunan, E.; Desiraju, G. R.; Klein, R. A.; Sadlej, J.; Scheiner, S.; Alkorta, I.; Clary, D. C.; Crabtree, R. H.; Dannenberg, J. J.; Hobza, P.; Kjaergaard, H. G.; Legon, A. C.; Mennucci, B.; Nesbitt, D. J.; Pure Appl. Chem. 2011, 83, 1637.

26. Latimer, W. M.; Rodebush, W. H.; J. Am. Chem. Soc. 1920, 42, 1419.

27. Kasper, C.; J. Am. Chem. Soc. 1931, 53, 2424.

28. Coulson, C. A.; Danielson, U.; Atk. Fys. 1954, 8, 246.

29. Tsubomura, H.; Bull. Chem. Soc. Jpn. 1954, 27, 445.

30. Nukasawa, K.; Tanaka, J.; Nagakura, S.; J. Phys. Soc. Jpn. 1953, 8,792 .

31. Koski, W. S.; J. Am. Chem. Soc. 1960, 82, 4120.

32. Kollman, P. A.; J. Am. Chem. Soc. 1972, 94, 1837.

33. Koch, U.; Popelier, P. L. A.; J. Phys. Chem. A 1995, 99, 9747.

34. Hobza, P.; Annu. Rep. Prog. Chem., Sect. C 2004, 100, 3.

35. Del Bene, J. E.; J. Phys. Chem. A 2007, 111, 5509.

36. Grabowski, S. J.; Chem. Rev. 2011, 111, 2597.

37. Yang, Y.; Meuwly, M.; J. Chem. Phys. 2010, 133, 064503; Buckingham, A. D.; Fowler, P. W.; Hutson, J. M.; Chem. Rev. 1988, 88, 963; Cordeiro, M. A. M.; Cordeiro, J. M. M.; J. Braz. Chem. Soc. 2004, 15, 351

38. Pendás, A. M.; Blanco, M. A.; Francisco, E.; J. Chem. Phys. 2006, 125, 184112.

39. Blanco, M. A.; Pendás, A. M.; Francisco, E.; J. Chem. Theor. Comput. 2005, 1, 1096

40. Oliveira, B. G.; Araújo, R. C. M. U.; Carvalho, A. B.; Ramos, M. N.; Hernandes, M. Z.; Cavalcante, K. R.; J. Mol. Struct. (THEOCHEM) 2007, 802, 91.

41. Perrin, C. L.; Nielson, J. B.; Ann. Rev. Phys. Chem. 1997, 48, 511; Meot-Ner, M.; Chem. Rev. 2005, 105, 213; Kojić-Prodi, B.; Molćanov, K.; Acta Chim. Slov. 2008, 55, 692; Arunan, E.; Curr. Sci. 1999, 77, 1233; Rustici, V. C. F.; Caramori, G. F.; Galembeck, S. E.; Quim. Nova 2006, 29, 1187; Rodrigues, J. A. R.; Quim. Nova 2000, 23, 812; Thar, J.; Kirchner, B.; J. Phys. Chem. A 2006, 110, 4229.

42. Oliveira, B. G.; Ramos, M. N.; Int. J. Quantum. Chem. 2010, 110, 307.

43. Grabowski, S. J.; Sokalski, W. A.; Leszczyński, J.; Chem. Phys. Lett. 2006, 422, 334.

44. Brookhart, M.; Green, M. L. H.; Parkin, G.; Proc. Natl. Acad. Sci. U. S. A. 2007, 114, 6908.

45. Oliveira, B. G.; Araújo, R. C. M. U.; Leite, E. S.; Ramos, M. N.; Int. J. Quantum. Chem. 2011, 111, 111; Palusiak, M.; Grabowski, S. J.; Struct. Chem. 2008, 19, 5.

46. Mohajeri, A.; Alipour, M.; Mousaee, M.; J. Phys. Chem. A 2011, $115,4457$.

47. Parthasarathi, R.; Subramanian, V.; Struct. Chem. 2005, 16, 243.

48. Capim, S. L.; Santana, S. R.; Oliveira, B. G.; Rocha, G. B.; Vasconcellos, M. L. A. A.; J. Braz. Chem. Soc. 2010, 21, 1718; Wheeler, S. E.; J. Am. Chem. Soc. 2011, 133, 10262; Woo, S.; Rothemund, P. W. K.; Nat. Chem. 2011, 3, 620 .

49. Lipkowski, P.; Grabowski, S. J.; Leszczyński, S. J.; J. Phys. Chem. A 2006, 110, 10296; Grabowski, S. J.; J. Phys. Chem. A 2011, 115, 12340.

50. Davies, R. H.; Mason, R. C.; Smith, D. A.; McNeillie, D. J.;
James, R.; Int. J. Quantum. Chem. 1978, 14, 221; Chiou, J. S.; Ma, S. M.; Kamaya, H.; Ueda, I.; Science 1990, 248, 583; Abraham, M. H.; Lieb, W. R.; Franks, N. P.; J. Pharm. Sci. 1991, 80, 719; Sándorfy, C.; J. Mol. Struct. 2004, 708, 3.

51. Braun, C. L.; Smirnov, S. N.; J. Chem. Educ. 1993, 70, 612.

52. Autumn, K.; Liang, Y. A.; Hsieh, S. T.; Zesch, W.; Chan, W. P.; Kenny, T. W.; Fearing, R.; Full, R. J.; Nature 2000, 405, 681; Autumn, K.; Sitti, M.; Liang, Y. A.; Peattie, A. M.; Hansen, W. R.; Sponberg, S.; Kenny, T. W.; Fearing, R.; Israelachvili, J. N.; Full, R. J.; Proc. Natl. Acad. Sci. U. S. A. 2002, 99, 12252.

53. Fernandez, J. A.; Jockers, K.; Rep. Prog. Phys. 1983, 46, 665; Whipple, F. L.; Huebner, W. F.; Ann. Rev. Astron. Astrophys. 1976, 14, 143.

54. Lemes, N. H. T.; Oliveira, J. M.; Braga, J. P.; Quim. Nova 2010, 33,1325

55. van Helden, A.; Willem Hendrik Keesom 1876 - 1956: A History of Science in the Netherlands, Survey, Themes and Reference, Leiden: Brill, 1999.

56. Reiding, J.; Ambix 2010, 57, 275; Arunan, E.; J. Sci. Educ. 2010, $14,667$.

57. London, F.; Z. Physik 1930, 63, 245; London, F.; Trans. Faraday Soc. $1937,33,8$.

58. Kamiya, M.; Tsuneda, T.; Hirao, K.; J. Chem. Phys. 2002, 117, 6010.

59. Ben-Avraham, D.; J. Stat. Phys. 2006, 123, 709; Rose, G. D.; Wolfenden, R.; Annu. Rev. Biophys. Biomol. Struct. 1993, 22, 381; Kuhn, B.; Mohr, P.; Stahl, M.; J. Med. Chem. 2010, 53, 2601; Desiraju, G. R.; Cryst. Growth Des. 2011, 11, 896.

60. Duvoisin Jr., S.; Lima, I. C. V.; Kuhnen, C. A.; Quim. Nova 2011, 34,1591

61. van Oss, C. J.; Good, R. J.; Chaudhury, M. K.; J. Colloid Interface Sci. 1986, 111, 378.

62. Steiner, T.; Desiraju, G. R.; Chem. Commun. 1998, 891

63. Rank, J. A.; Baker, D.; Biophys. Chem. 1998, 71, 199.

64. Bian, L.; J. Phys. Chem. A 2003, 107, 11517.

65. Hammerum, S.; J. Am. Chem. Soc. 2009, 131, 8627

66. Sobczyk, L.; Grabowski, S. J.; Krygowski, T. M.; Chem. Rev. 2005, 105, 3513 .

67. Lukin, O.; Leszczyński, J.; J. Phys. Chem. A 2002, 106, 6775.

68. Lennard-Jones, J. E.; Proc. R. Soc. 1924, 106, 463; Araujo, J. C. B.; Borges, G. R. P.; Drigo Filho, E.; Rev. Bras. Ensino Fís. 2006, 28 , 41; Levine, I. N.; Quantum Chemistry, Prentice-Hall: New Jersey, 2001.

69. Hagler, A. T.; Lifson, S.; Dauber, P.; J. Am. Chem. Soc. 1979, 101, 5122 .

70. Kaplan, I. G.; Theory of Molecular Interactions; Studies in Physical and Theoretical Chemistry, Elsevier: Amsterdam, 1986; Herbert, J. M.; Jacobson, L. D.; Lao, K. U.; Rohrdanz, M. A.; Phys. Chem. Phys. Chem. 2012, 14, 7679 .

71. Bleiholder, C.; Werz, D. B.; Köppel, H.; Gleiter, R.; J. Am. Chem. Soc. 2006, 128, 2666; Gleiter, R.; Gygax, R.; Top. Curr. Chem. 1976, $63,49$.

72. Pauling, L.; The Nature of the Chemical Bond, Cornell University Press: Ithaca, 1939; Pauling, L.; J. Chem. Educ. 1992, 69, 519; Pimentel, G. C.; McClellan, A. L.; The Hydrogen Bond, W. H. Freeman: San Francisco, 1960

73. Desiraju, G. R.; Angew. Chem., Int. Ed. 2010, 49, 2.

74. Johnson, E. R.; Keinan, S.; Mori-Sánchez, P.; Contreras-García, J.; Cohen, A. J.; Yang, W.; J. Am. Chem. Soc. 2010, 132, 6498.

75. Bondi, A.; J. Phys. Chem. 1964, 68, 441.

76. Hill, J. W.; J. Chem. Educ. 1986, 63, 503.

77. Allen, L. C.; J. Am. Chem. Soc. 1975, 97, 6921.

78. van Duijneveldt, F. B.; Murrell, J. N.; J. Chem. Phys. 1967, 46, 1759 . 
79. Jeziorski, B.; Moszynski, R.; Szalewicz, K.; Chem. Rev. 1994, 94, 1887.

80. Nagase, S.; Fueno, T.; Theor. Chem. Acc. 1974, 35, 217.

81. Sinnokrot, M. O.; Sherrill, C. D.; J. Phys. Chem. A 2006, 110, 10656; Hohenstein, E. G.; Sherrill, C. D.; WIREs Comput. Mol. Sci. 2012, 2, 304.

82. Alkorta, I.; Blanco, F.; Elguero, J.; J. Phys. Chem. A 2008, 112, 6753; Gao, Y.; Zhang, L.; Wang, Y.; Li, H.; J. Phys. Chem. B 2010, 114, 2828.

83. Ottiger, P.; Pfaffen, C.; Leist, R.; Leutwyler, S.; Bachorz, R. A.; Klopper, W.; J. Phys. Chem. B 2009, 113, 2937; D’Oria, E.; Novoa, J. J.; J. Phys. Chem. A 2011, 17, 13114; Majerz, I.; Org. Biomol. Chem. 2011, 9, 1466.

84. Ma, J. C.; Dougherty, D. A.; Chem. Rev. 1997, 97, 1303.

85. Umeyama, H.; Morokuma, K.; J. Am. Chem. Soc. 1977, 99, 1316.

86. Lin, K.-J.; Cheng, M.-C.; Wang, Y.; J. Phys. Chem. 1994, 98, 11685 .

87. Lima, A. N.; J. Chem. Phys. 2010, 132, 014110.

88. Chandra, A. K.; Nguyen, M. T.; J. Phys. Chem. A 1998, 102, 6865.

89. Hesselmann, A.; J. Phys. Chem. A 2011, 115, 11321; Sadlej, J.; Moszynski, R.; Dobrowolski, J. C.; Mazurek, A. P.; J. Phys. Chem. A 1999, 103, 8528 .

90. Oliveira, B. G.; Araújo, R. C. M. U.; Quim. Nova 2007, 30, 791; Oliveira, B. G.; Araújo, R. C. M. U.; Ramos, M. N.; Quim. Nova 2010, $33,1155$.

91. Oliveira, B. G.; Araújo, R. C. M. U.; Can. J. Chem. 2012, 90, 368

92. Oliveira, B. G.; Araújo, R. C. M. U.; Carvalho, A. B.; Ramos, M. N.; J. Mol. Model. 2011, 17, 2847; Humbel, S.; J. Phys. Chem. A 2002, 106, 5517.

93. Dannenberg, J. J.; Haskamp, L.; Masunov, A.; J. Phys. Chem. A 1999, 103, 7083; Isaacs, E. D.; Shukla, A.; Platzman, P. M.; Hamann, D. R.; Barbiellini, B.; Tulk, C. A.; Phys. Rev. Lett. 1999, 82, 600; Jacobsen, H.; Dalton Trans. 2010, 39, 5426.

94. Kenny, P. W.; J. Chem. Inf. Model. 2009, 49, 1234

95. Wu, Q.; Yang, W.; J. Chem. Phys. 2002, 116, 515.

96. Karthikeyan, S.; Sedlak, R.; Hobza, P.; J. Phys. Chem. A 2011, $115,9422$.

97. Chałasiński, G.; Szczę́śniak, M. M.; Chem. Rev. 1994, 94, 1723.

98. Heerdt, G.; Morgon, N. E.; Quim. Nova 2011, 34, 868

99. Møller, C.; Plesset, M. S.; Phys. Rev. 1934, 46, 618.

100. Sherrill, C. D.; Schaefer III, H. F.; Adv. Quantum Chem. 1999, 34, 143.

101. Shavitt, I.; Bartlett, R. J.; Many-Body Methods in Chemistry and Physics: MBPT and Coupled-Cluster Theory, Cambridge University Press: Cambridge, 2009.

102. Morgon, N. E.; Custodio, R.; Quim. Nova 1995, 18, 44.

103. Geerlings, P.; De Proft, F.; Langenaeker, W.; Chem. Rev. 2000, 103,1793

104. Zhao, Y.; Truhlar, D. G.; Acc. Chem. Res. 2008, 41, 157.

105. van der Vaart, A.; Merz Jr., K. M.; J. Phys. Chem. A 1999, 103, 3321.

106. Su, P.; Li, H.; J. Chem. Phys. 2009, 131, 014102.

107. http://www.physics.udel.edu/ szalewic/SAPT, acessada em Outubro 2011.

108. Schrödinger, E.; Ann. der Physik 1926, 79, 734; Custodio, R.; Politi, J. R. S.; Segala, M.; Haiduke, R. L. A.; Quim. Nova 2002, 25, 159; Hermoso, W.; Ornellas, F. R.; Quim. Nova 2009, 32, 2487; Popelier, P. L. A.; Solving the Schrödinger Equation: Has Everything Been Tried?, Imperial College Press: London, 2011.

109. Lotrich, V. F.; Szalewicz, K.; J. Chem. Phys. 1997, 106, 9668.

110. Lotrich, V. F.; Szalewicz, K.; J. Chem. Phys. 2000, 112, 112.

111. Riley, K. E.; Hobza, P.; J. Chem. Theory Comput. 2008, 4, 232

112. Stone, A. J.; Misquitta, A. J.; Chem. Phys. Lett. 2009, 473, 201.
113. Hoffman, G. G.; J. Chem. Educ. 2005, 82, 1418

114. Raghavachari, K.; Anderson, J. B.; J. Phys. Chem. 1996, 100, 12960.

115. Korona, T.; J. Chem. Theory Comput. 2009, 5, 2663.

116. Podeszwa, R.; Szalewicz, K.; J. Chem. Phys. 2012, 136, 161102; Rezáč, J.; Hobza, P.; J. Chem. Theory Comput. 2011, 7, 685.

117. Podeszwa, R.; Szalewicz, K.; J. Chem. Phys. 2007, 126, 194101.

118. Misquitta, A. J.; Podeszwa, R.; Jeziorski, B.; Szalewicz, K.; J. Chem. Phys. 2005, 123, 214103; Hesselmann, A.; Korona, T.; Phys. Chem. Phys. Chem. 2011, 14, 732.

119. Szalewicz, K.; Jeziorski, B.; Molecular Interactions - from van der Waals to strongly bound complexes, Wiley: New York, 1997.

120. Kruse, H.; Grimme, S.; J. Chem. Phys. 2012, 136, 154101.

121. Jeziorski, B.; Moszyski, R.; Ratkiewicz, A.; Rybak, S.; Szalewicz, K.; Williams, H. L.; Clementi, E.; Methods and Techniques in Computational Chemistry: METECC-94, vol. B, STEF: Cagliari, 1993.

122. Scheiner, S.; J. Chem. Phys. 2011, 134, 164313; Scheiner, S.; Phys. Chem. Chem. Phys. 2011, 13, 13860; Scheiner, S.; J. Chem. Phys. 2011, 134, 094315 .

123. Boys, S. F.; Bernardi, F.; Mol. Phys. 1970, 19, 553; van Duijneveldt, F. B.; van Duijneveldt-van de Rijdt, J. G. C. M.; van Lenthe, J. H.; Chem. Rev. 1994, 94, 1873

124. Barone, V.; J. Chem. Phys. 2004, 120, 3059; Govender, A.; Ferré, D. C.; Niemantsverdriet, J. W.; Chem. Phys. Chem. 2012, 13, 1591

125. Reinhardt, P.; Piquemal, J.-P.; Int. J. Quantum Chem. 2009, 109, 3259 .

126. Panek, J. J.; Jezierska, A.; J. Phys. Chem. A 2007, 111, 650.

127. Araújo, R. C. M. U.; Soares, V. M.; Oliveira, B. G.; Lopes, K. C.; Ventura, E.; Monte, S. A.; Santana, O. L.; Carvalho, A. B.; Ramos, M. N.; Int. J. Quantum Chem. 2006, 106, 2714; King, B. F.; Weinhold, F.; J. Chem. Phys. 1995, 103, 333

128. Oliveira, B. G.; Araújo, R. C. M. U.; Soares, V. M.; Ramos, M. N.; J. Theor. Comput. Chem. 2008, 7, 247; Zhao, Q.; Feng, D.; Hao, J.; J. Mol. Model. 2011, 17, 2817.

129. Sánchez, M.; Provasi, P. F.; Aucar, G. A.; Alkorta, I.; Elguero, J.; J. Phys. Chem. B 2005, 109, 18189; Grabowski, S. J.; Bilewicz, E.; Chem. Phys. Lett. 2006, 427, 51.

130. Rivelino, R.; Chaudhuri, P.; Canuto, S.; J. Chem. Phys. 2003, 118, 10593.

131. Estarellas, C.; Frontera, A.; Quiñonero, D.; Chem. Phys. Chem. 2011, 12, 2742

132. Parra, R. D.; Bulusu, S.; Zeng, X. C.; J. Chem. Phys. 2003, 118, 3499.

133. Bauer, M.; Spange, S.; Angew. Chem., Int. Ed. 2011, 50, 9727.

134. Hobza, P.; Zahradník, R.; Chem. Rev. 1988, 88, 871.

135. Rocha, W. R.; Química Nova na Escola 2001, Caderno Temático N. 4, 31; Morgon, N. H.; Soares, A. G.; Quim. Nova 1998, 21, 259; Pliego Jr., J. R.; Almeida, W. B.; Quim. Nova 1997, 20, 146.

136. Williams, H. L.; Korona, T.; Bukowski, R.; Jeziorski, B.; Sz, K.; Chem. Phys. Lett. 1996, 262, 431; Jeziorska, M.; Bukowski, R.; Cencek, W.; Jaszunski, M.; Jeziorski, B.; Sz, K.; Coll. Czech. Chem. Commun. 2003, 68, 463.

137. Tsuzuki, S.; Lüthi, H. P.; J. Chem. Phys. 2001, 114, 3949; Dion, M.; Rydberg, H.; Schroeder, E.; Langreth, D. D.; Lundqvist, B. I.; Phys. Rev. Lett. 2004, 92, 246401.

138. Panek, J. J.; Wawrzyniak, P. K.; Latajka, Z.; Lundell, J.; Chem. Phys. Lett. 2006, 417, 100; Doran, M. D.; J. Phys. B: At., Mol. Opt. Phys. 1974, 7, 558 .

139. Khriachtchev, L.; Räsänen, M.; Gerber, R. B.; Acc. Chem. Res. 2009, 42, 183 .

140. Cukras, J.; Sadlej, J.; Chem. Phys. Lett. 2008, 459, 44.

141. Kim, K. S.; Tarakeshwar, P.; Lee, J. J.; Chem. Rev. 2000, 100, 4145 . 
142. Jaeger, H. M.; Schaefer, H. F.; Hohenstein, E. G.; Sherrill, C. D.; Comput. Theor. Chem. 2011, 973, 47; Tarakeshwar, P.; Kim, K. S.; J. Mol. Struct. 2002, 615, 227.

143. Cybulski, H.; Sadlej, J.; J. Chem. Theory Comput. 2008, 4, 892.

144. Munusamy, E.; Sedlak, R.; Hobza, P.; Chem. Phys. Chem. 2011, $12,3253$.

145. Rzepkowska, J.; Uras, N.; Sadlej, J.; Buch, V; J. Phys. Chem. A 2002, 106, 1790.

146. Hesselmann, A.; Jansen, G.; Schütz, M.; J. Chem. Phys. 2005, $122,014103$.

147. Podeszwa, R.; Szalewicz, K.; Chem. Phys. Lett. 2005, 412, 488.

148. Murthy, A. S. N.; Rao, C. N. R.; Appl. Spectrosc. Rev. 1968, 2, 69.

149. Araújo, R. C. M. U.; Ramos, M. N.; J. Mol. Struct. (THEOCHEM) 1996, 366, 233; Araújo, R. C. M. U.; Ramos, M. N.; Spectrochim. Acta A 1995, 51, 821; Yu, W.; Lin, Z.; Huang, Z.; Chem. Phys. Chem. 2006, 7, 828 .

150. Hobza, P.; Havlas, Z.; Chem. Rev. 2000, 100, 4253; Murray, J.; Concha, M. C.; Lane, P.; Hobza, P.; Politzer, P.; J. Mol. Model. 2008, 14, 699; Joseph, J.; Jemmis, E. D.; J. Am. Chem. Soc. 2007, 129, 4620.

151. Oliveira, B. G.; Araújo, R. C. M. U.; Ramos, M. N.; J. Mol. Struct. (THEOCHEM) 2010, 944, 168; Barnes, A. J.; J. Mol. Struct. 2004, 704, 3.

152. Li, X.; Liu, L.; Schlegel, H. B.; J. Am. Chem. Soc. 2002, 124, 9639.

153. Hermansson, K.; J. Phys. Chem. A 2002, 106, 4695.

154. Nesbitt, D. J.; Chem. Rev. 1988, 88, 843; Perera, L.; Amar, F. G.; J. Chem. Phys. 1990, 93, 4884; Grandinetti, F.; Eur. J. Mass Spectrom. 2011, 17, 423.

155. Hobza, P.; Spirko, V.; Selzle, H. L.; Schlag, E. W.; J. Phys. Chem. 1998, 102, 2501.

156. Reimann, B.; Buchfold, K.; Vaupel, S.; Brutschy, B.; Havlas, Z.; Spirko, V.; Hobza, P.; J. Phys. Chem. A 2001, 105, 5560.

157. Zierkiewicz, W.; Michalska, D.; Zeegers-Huyskens, T.; Phys. Chem. Chem. Phys. 2010, 12, 13681; Wang, S.-C.; Sahu, P. K.; Lee, S.-L; Chem. Phys. Lett. 2005, 406, 143; Hobza, P.; Havlas, Z.; Theor. Chem. Acc. 2002, 108, 325.

158. Szalewicz, K.; WIREs Comput. Mol. Sci 2012, 2, 254.

159. Parreira, R. L. T.; Caramori, G. F.; Morgon, N. H.; Galembeck,
S. E.; Int. J. Quantum Chem. 2012, 112, 1401; Waller, M. P.; Kruse, H.; Muck-Lichtenfeld, C.; Grimme, S.; Chem. Soc. Rev. 2012, 41, 3119; Wilson, A. J.; Nat. Chem. 2011, 3, 193.

160. Noodleman, L.; Lovell, T.; Han, W.-G.; Liu, T.; Torres, R.; Himo, F.; Comp. Coord. Chem. 2004, 2, 491; Kohn, W.; Hohenberg, P.; Phys. Rev. 1964, 136, B864.

161. Parr, R. G.; Ayers, P. W.; Nalewajski, R. F.; J. Phys. Chem. A 2005, 109, 3957; Matta, C. F.; Bader, R. F. W.; J. Phys. Chem. A 2006, 110,6365 .

162. Bader, R. F. W; Atoms in Molecules. A Quantum Theory, Oxford University Press: Oxford, 1990; Bader, R. F. W.; Chem. Rev. 1991, 91, 893.

163. Bader, R. F. W.; Nguyen-Dang, T. T.; Adv. Quantum Chem. 1981, $14,63$.

164. Bader, R. F. W.; Adv. Quantum Chem. 2009, 57, 285.

165. Schwinger, J.; Phys. Rev. 1951, 82, 914; Schwinger, J.; Phys. Rev. 1953, 91,713 .

166. Cremer, D.; Kraka, E.; J. Am. Chem. Soc. 1985, 107, 3811.

167. Fuster, F.; Grabowski, S. J.; J. Phys. Chem. A 2011, 115, 10078.

168. Grabowski, S. J.; Sokalski, W. A.; Dyguda, E.; Leszczyński, J.; J. Phys. Chem. B 2006, 110, 6444; Grabowski, S. J.; Sokalski, W. A.; Leszczyński, J.; J. Chem. Phys. 2007, 337, 68; Grabowski, S. J.; J. Phys. Chem. A 2012, 116, 1838.

169. Rozas, I.; Alkorta, I.; Elguero, J.; J. Phys. Chem. A 1997, 101, 9457.

170. Szatyłowicz, H.; J. Phys. Org. Chem. 2008, 21, 897.

171. Rivelino, R.; Canuto, S.; Chem. Phys. Lett. 2000, 322, 207; Grabowski, S. J.; Chem. Phys. Lett. 2001, 338, 361; Wojtulewski, S.; Grabowski, S. J.; J. Mol. Struct. 2002, 605, 235; Wojtulewski, S.; Grabowski, S. J.; Chem. Phys. Lett. 2003, 378, 388; Fileti, E. E.; Chaudhuri, P.; Canuto, S.; Chem. Phys. Lett. 2004, 400, 494

172. Oliveira, B. G.; Leite, L. F. C. C.; J. Mol. Struct. (THEOCHEM) 2009, 915, 38.

173. Rozas, I.; Alkorta, I.; Elguero, J.; J. Phys. Chem. A 1997, 101, 4236; Oliveira, B. G.; Araújo, R. C. M. U.; J. Mol. Model. 2012, 18, 2845; Karalti, O.; Alfè, D.; Gillan, M. J.; Jordan, K. D.; Phys. Chem. Chem. Phys. 2012, 14, 7846. Podeszwa, R.; Cencek, W.; Szalewicz, K.; J. Chem. Theory Comput. 2012, 8, 1963. 